\title{
Bead lightning formation
}

\author{
G. O. Ludwig \\ Associated Plasma Laboratory, National Space Research Institute, 12227-010, São José dos Campos, \\ SP, Brazil
}

M. M. F. Saba

Division of Space Geophysics, National Space Research Institute, 12227-010, São José dos Campos, SP, Brazil

(Received 13 June 2005; accepted 11 August 2005; published online 21 September 2005)

\begin{abstract}
Formation of beaded structures in triggered lightning discharges is considered in the framework of both magnetohydrodynamic (MHD) and hydrodynamic instabilities. It is shown that the space periodicity of the structures can be explained in terms of the kink and sausage type instabilities in a cylindrical discharge with anomalous viscosity. In particular, the fast growth rate of the hydrodynamic Rayleigh-Taylor instability, which is driven by the backflow of air into the channel of the decaying return stroke, dominates the initial evolution of perturbations during the decay of the return current. This instability is responsible for a significant enhancement of the anomalous viscosity above the classical level. Eventually, the damping introduced at the current channel edge by the high level of anomalous viscous stresses defines the final length scale of bead lightning. Later, during the continuing current stage of the lightning flash, the MHD pinch instability persists, although with a much smaller growth rate that can be enhanced in a M-component event. The combined effect of these instabilities may explain various aspects of bead lightning.
\end{abstract}

(C) 2005 American Institute of Physics. [DOI: 10.1063/1.2048907]

\section{INTRODUCTION}

A not well understood aspect of lightning discharges is the beaded appearance of the decaying lightning channel glow, an event rarely observed in natural lightning but usually observed in triggered lightning experiments. ${ }^{1-6}$ In a recent book, Rakov and Uman ${ }^{6}$ pointed out that bead lightning clearly involves an already existing lightning channel, but there is no consensus view of the physical mechanism for its formation. This periodic structure has been recently recorded using a fast charge-coupled-device (CCD) camera during a series of triggered lightning experiments carried out in the International Center for Triggered and Natural Lightning in Cachoeira Paulista, SP, Brazil. ${ }^{7}$ Small rockets carrying a thin copper wire connected to a launching platform artificially triggered 23 flashes during the past 5 years. Figure 1 presents a sequence of pictures taken at 1000 frames per second showing the slow cooling stage of the discharge channel between the last two consecutive strokes of a ten-stroke flash.

Typically, the current in the return stroke of a lightning discharge attains the median value of 30-40 kA in $1 \mu$ s or less and decays with a time constant 20-60 $\mu$ s to a continuing current component during pauses between successive strokes. ${ }^{4}$ Low currents of the order of 100 A may flow for periods from milliseconds to tens of milliseconds after each return stroke. ${ }^{5}$ Each successive stroke is formed only after the continuing current terminates with a typical $50 \mathrm{~ms}$ duration. At this time the channel has cooled down and its linear resistance has increased to levels sufficiently large to allow the excitation of a new ionization wave, which leads to a new stroke.

The peak current measured during the triggered lightning shown in Fig. 1 is $45 \mathrm{kA}$ with a typical wave form as shown in Fig. 2. In this case, the strong and fast initial peak is followed by a smoothly decaying continuing current. The last frame in Fig. 1 shows the start of the last return stroke of the ten-stroke flash, which ends on the tip of a Franklin lightning rod that can be seen on the right-hand side of the sequence of pictures. The images clearly show the beaded structure during pauses in triggered lightning discharges. The relevant information obtained from these pictures is the scale of the beads, which corresponds to a characteristic axial wavelength of about $0.5 \mathrm{~m}$. This length scale is estimated from the known dimensions of the launching platform that can be seen in some of the frames. It seems that the instability associated with the formation of the beads covers a wide range of wavelengths, starting from very small values and ending at wavelengths near $0.5 \mathrm{~m}$. This particular wavelength has a preeminent role in the last stages of the discharge evolution. The time scale of the instability is very short, and most of the development takes place during the first few milliseconds, when some of the pictures are unfortunately saturated by light.

Most of the proposed bead lightning models ${ }^{1,6,8-10}$ describe optical, pinch and luminous effects that depend on geometric distortions of the lightning channel with respect to the viewing direction or on periodic modulation of the channel properties as a function of height. However, these models neither explain how the lightning channel becomes distorted or modulated, nor predict the spatial scale of the structure. Under laboratory conditions, Tumakaev ${ }^{11}$ observed the fragmentation of high-voltage glow discharges at atmospheric pressure and hypothesized bead lightning as a stratification process. But, the low currents of $10-30 \mathrm{~mA}$ and high voltages of the experiment, of $50-60 \mathrm{kV}$ across a $1 \mathrm{M} \Omega$ ballast 

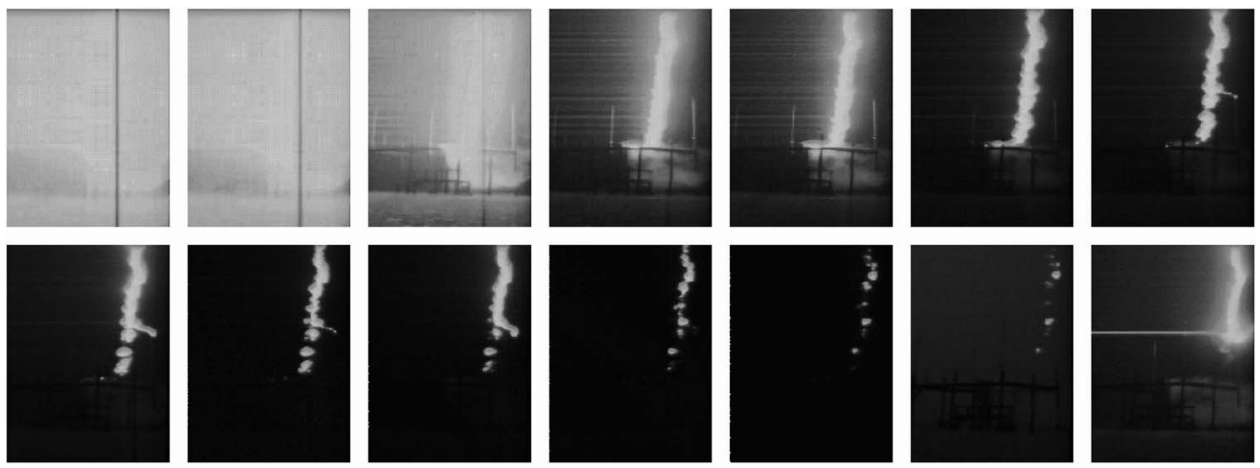

FIG. 1. Beaded structure in a triggered lightning discharge shown at $1 \mathrm{~ms}$ time intervals.

resistance applied to an interelectrode gap $15 \mathrm{~cm}$ long, are incompatible with the conditions expected in the inter stroke period in a lightning flash, that is, electric fields of about $500-800 \mathrm{~V} / \mathrm{m}$ for quasisteady currents of the order of $100 \mathrm{~A}$, with fast decreasing electric field values for currents below about $10 \mathrm{~A} .{ }^{12}$ Nevertheless, a process of self-organization of the discharge involving thermal effects cannot be disregarded.

The purpose of this article is to consider the role of both the magnetohydrodynamic (MHD) and the hydrodynamic instabilities in the formation of the beaded structure in triggered lightning discharges. First, consider the characteristics of the pinch effect, which has been proposed by $\mathrm{Uman}^{8}$ as a possible cause for bead lightning. This effect has been recently shown ${ }^{13}$ to give the correct range of wavelengths to explain the beaded structure if one takes into account the anomalous character of the discharge viscosity. However, it was also concluded that the MHD (pinch) instabilities are too weak to explain the appearance of the beaded structure during the short interval of decay of the return current, of the order of $300 \mu$ s or less in the case of Fig. 2. This conclusion is in agreement with the discussion presented by Tumakaev ${ }^{11}$ about the pinch effect. It was then put forward the need to include a transient radial acceleration in the model to account for the effects of expansion and contraction of the discharge quasiequilibrium during the decay of the current. ${ }^{13}$ As pointed out both by Hill ${ }^{14}$ and Aleksandrov et al. ${ }^{12}$, during
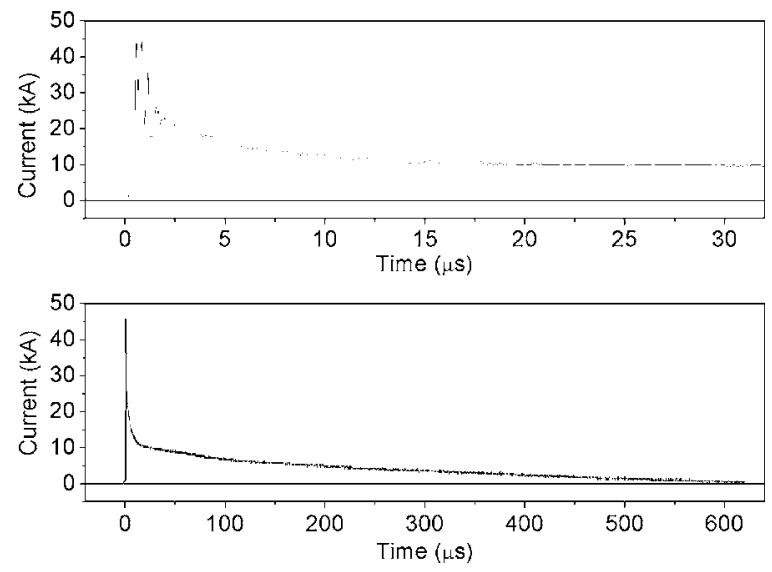

FIG. 2. Typical current wave form of a return stroke in triggered lightning discharges shown in two different time scales. the contraction of air into the channel the situation is favorable to the onset of the hydrodynamic Rayleigh-Taylor instability in a thin layer near the boundary. Taking these effects into account, the present paper shows that both the MHD and hydrodynamic instabilities have basically the same spatial structure, resulting in deformations on spatial scales compatible with the ones estimated from the images shown in Fig. 1 . But, the radial acceleration has a stabilizing effect during the initial expansion stage ( $t \lesssim 100 \mu \mathrm{s}$ in the case of Fig. 2$)$, when a large current is flowing through a small diameter channel and the MHD instabilities are relatively strong, that is, the acceleration impedes the pinching of the initially growing current channel. On the other hand, during the latter contraction stage $(t \gtrsim 100 \mu \mathrm{s})$ the discharge becomes unstable to both the MHD and hydrodynamic perturbations. At this stage, the current decayed to a low level and the channel radius is relatively large, so that the MHD instability is weak and the hydrodynamic instability dominates the subsequent evolution. The hydrodynamic Rayleigh-Taylor instability has a very fast growth rate and produces turbulent fluid motion which enhances the viscosity inside the discharge significantly above the classical level. It is the expected high level of anomalous viscosity that results in the characteristic structure of bead lightning. ${ }^{15}$ After a few characteristic times $(t$ $\gtrsim 1 \mathrm{~ms}$ ) the hot gas in the channel starts expanding and cooling down with anomalously high diffusivity and thermal conductivity. Concurrently, the onset of cold air backflow into the channel occurs later and later at larger radial distances, as also pointed out by $\mathrm{Hill}^{14}$ in the case of a spark channel, moving the region of instability outwards for a few milliseconds over an already deformed channel. After a total time of about $5 \mathrm{~ms}$, according with Fig. 1, the air backflow in the channel region ceases, quenching the Rayleigh-Taylor instability. At this time the lightning flash is well into the continuing current regime ${ }^{5}$ that lasts from several tens to hundreds of milliseconds, and the MHD instabilities persist, although with a very small growth rate. During this stage, these instabilities may be significant only in the occurrence of a $\mathrm{M}$ component, which are current pulses with a duration of $100-1000 \mu$ s and amplitudes generally smaller than those of the return strokes. In general, the MHD instabilities are much weaker than the hydrodynamic instability whereas this one lasts, having a much smaller effect in defining the structure of bead lightning. 
A simplified dispersion relation for the MHD and hydrodynamic instabilities in the cylindrical geometry of a lightning discharge is derived in Sec. II. The model includes both the electromagnetic effects and the forces associated with the radial acceleration of air at the current channel. It is assumed that the acceleration varies on a much longer time scale than the instability, so that the acceleration can be taken as approximately constant in the model. Further, the model includes viscosity effects, which are essential in defining the scale length of the instability. A discussion of the results is presented in Sec. III. There, the lightning parameters are estimated using simple analytic formulas based on standard shock and compression wave theories. In order to relate the results of previous analyses and simulations, and to unify the notation, a review of these theories is presented in the appendices under a consistent formulation. Finally, Sec. IV presents the conclusions.

\section{INSTABILITY OF THE LIGHTNING CHANNEL BOUNDARY}

In the following, both the MHD (pinch) and the hydrodynamic (Rayleigh-Taylor) instabilities in a cylindrical discharge are examined by including the effects of the magnetic field and of the transient radial acceleration of air at the channel boundary. It will be shown that the observed spatial scale of the beaded structure can be explained in terms of these instabilities if the anomalous character of air viscosity in the discharge is taken into account. The damping introduced by the anomalous viscous stresses at the edge of the channel defines the spatial scale of the unstable freeboundary modes in the decaying lightning discharge.

The problems examined in this article are described by the fluid equations for a viscous magnetized plasma. These are the mass conservation equation, Navier-Stokes equation, local equation of state, Ampère's law, and combined Faraday's and Ohm's laws for a perfectly conductive fluid, given respectively by

$$
\begin{aligned}
& \frac{\partial \rho}{\partial t}+\nabla \cdot(\rho \mathbf{v})=0, \\
& \rho \frac{d \mathbf{v}}{d t}=-\nabla p+\mathbf{j} \times \mathbf{B}+\eta \nabla^{2} \mathbf{v}+\frac{1}{3} \eta \nabla(\nabla \cdot \mathbf{v})+\rho \mathbf{a}, \\
& \frac{d}{d t}\left(\frac{p}{\rho^{\gamma}}\right)=0, \\
& \nabla \times \mathbf{B}=\mu_{0} \mathbf{j}, \\
& \frac{\partial \mathbf{B}}{\partial t}=\nabla \times(\mathbf{v} \times \mathbf{B}) .
\end{aligned}
$$

The fluid variables are the mass density $\rho$, the fluid velocity $\mathbf{v}$, and the pressure $p$. The electromagnetic variables are the current density $\mathbf{j}$ and the magnetic flux density $\mathbf{B}$. The coefficient of shear viscosity $\eta$ is assumed uniform and the coefficient of volume viscosity has been neglected. Also, $\gamma$ $=c_{p} / c_{v}$ is the ratio of specific heats and $d / d t \equiv \partial / \partial t+\mathbf{v} \cdot \nabla$ is the convective derivative. The force density $\rho \mathbf{a}$ is included to describe the effect of fluid acceleration normal to the fluid interface.

The stability analysis is carried out for the radial expansion/contraction of a cylindrical column with total axial current $I$ flowing in the plasma inside the radius $r_{c}$, that is, the current channel of a lightning discharge. Assuming that the pressure makes a sharp transition, at $r=r_{c}$, from the constant value $p_{i}=p(0)$ inside the column to the value $p_{e}$ $=p(\infty)$ of the external gas pressure, the equilibrium relation for negligible flow is

$$
p_{i}+\bar{\rho} a L_{a}=p_{e}+\frac{\mu_{0} I^{2}}{8 \pi^{2} r_{c}^{2}},
$$

where the radial acceleration has a typical value $a$ and a scale length $L_{a}$ defined by

$$
\bar{\rho} a L_{a}=\frac{1}{r_{c}^{2}} \int_{0}^{\infty} \rho(r) a(r) r^{2} d r,
$$

and $\bar{\rho}$ is some average value of the density at the boundary, which rises from the internal value $\rho_{i}$ to the external value $\rho_{e}>\rho_{i}$. For example, a narrow acceleration distribution centered on $r_{c}$ with an abrupt change in the density corresponds to the average value $\bar{\rho}=\left(\rho_{e}+\rho_{i}\right) / 2$. In this case, the quantity $2 \pi r_{c} L_{a} \bar{\rho}$ gives the total mass inside the cylindrical shell of unit height, radius $r_{c}$, and thickness $L_{a} \ll r_{c}$ (snowplow model). Now, during the contraction stage of a lightning discharge the acceleration distribution is located outside the channel boundary, $\bar{\rho} \cong \rho_{e}$ and $a$ has a negative sign.

For a small perturbation in the fluid velocity, given in terms of the Lagrangian displacement $\boldsymbol{\xi}$ by $\delta \mathbf{v}=\partial \boldsymbol{\xi} / \boldsymbol{\partial} t$ $=-i \omega \xi$, the linearized equation of motion becomes

$$
\begin{gathered}
-\rho \omega^{2} \boldsymbol{\xi}-\frac{\mathbf{B}}{\mu_{0}} \cdot \nabla \delta \mathbf{B}=-\nabla \delta p^{*}-\mathbf{a} \nabla \cdot(\rho \boldsymbol{\xi})+\delta \mathbf{B} \cdot \frac{\nabla \mathbf{B}}{\mu_{0}} \\
-i \omega \eta \frac{4}{3} \nabla(\nabla \cdot \boldsymbol{\xi})+i \omega \eta \nabla \times(\nabla \times \boldsymbol{\xi}),
\end{gathered}
$$

where the total perturbed pressure is

$$
\delta p^{*}=\delta p+\frac{\mathbf{B}}{\mu_{0}} \cdot \delta \mathbf{B},
$$

and the internal magnetic field perturbation is

$$
\delta \mathbf{B}=\nabla \times(\boldsymbol{\xi} \times \mathbf{B})=-\mathbf{B}(\nabla \cdot \boldsymbol{\xi})+(\mathbf{B} \cdot \nabla) \boldsymbol{\xi}-(\boldsymbol{\xi} \cdot \nabla) \mathbf{B} .
$$

The external magnetic field perturbation is given in terms of a magnetic potential $\phi_{m}$ by

$$
\delta \mathbf{B}=-\mu_{0} \nabla \phi_{m},
$$

where $\nabla^{2} \phi_{m}=0$. For a compressive perturbation the perturbed pressure becomes

$$
\delta p=-\boldsymbol{\xi} \cdot \nabla p-\gamma p \nabla \cdot \boldsymbol{\xi} .
$$

Now, with $\hat{\mathbf{n}}$ denoting the unit normal to the interface and $\langle X\rangle$ the increment of $X$ across the interface, a set of appropriate boundary conditions is specified by the conditions of continuity of the magnetic field in the absence or presence of 
a surface current density $\mathbf{K}$, fluid continuity, no-slip, stress continuity, and pressure balance, given respectively by

$$
\begin{aligned}
& \langle\hat{\mathbf{n}} \cdot \mathbf{B}\rangle=0 \text { if } \mathbf{K}=0 \text { or } \hat{\mathbf{n}} \cdot[\mathbf{B}]_{r_{c}}=0 \text { if } \mathbf{K} \neq 0, \\
& \langle\hat{\mathbf{n}} \cdot \mathbf{v}\rangle=0, \\
& \langle\eta \hat{\mathbf{n}} \times \mathbf{v}\rangle=0, \\
& \langle\eta \hat{\mathbf{n}} \times[\hat{\mathbf{n}} \cdot(\nabla \mathbf{v})+(\nabla \mathbf{v}) \cdot \hat{\mathbf{n}}]\rangle=0, \\
& \left\langle p+\bar{\rho} \phi_{a}+\frac{B^{2}}{2 \mu_{0}}-2 \eta \hat{\mathbf{n}} \cdot(\nabla \mathbf{v}) \cdot \hat{\mathbf{n}}+\frac{2}{3} \eta \nabla \cdot \mathbf{v}\right\rangle=0,
\end{aligned}
$$

where $\bar{\rho}$ is the average mass density at the current channel edge and $\phi_{a}$ is the potential related to the acceleration by $\mathbf{a}$ $=-\nabla \phi_{a}$. The respective boundary conditions for the perturbations are

$$
\begin{aligned}
& \hat{\mathbf{n}} \cdot[\delta \mathbf{B}-\nabla \times(\boldsymbol{\xi} \times \mathbf{B})]_{r_{c}}=0 \text { if } \mathbf{K} \neq 0, \\
& \hat{\mathbf{n}} \cdot\langle\boldsymbol{\xi}\rangle=0, \\
& \hat{\mathbf{n}} \times\langle\boldsymbol{\xi}\rangle=0, \\
& \langle\eta \hat{\mathbf{n}} \times[\hat{\mathbf{n}} \cdot(\nabla \boldsymbol{\xi})+(\nabla \boldsymbol{\xi}) \cdot \hat{\mathbf{n}}]\rangle=0, \\
& \left\langle\delta p^{*}+\boldsymbol{\xi} \cdot \nabla p-\left(\bar{\rho} \phi_{a}+\frac{2}{3} i \omega \eta\right) \nabla \cdot \boldsymbol{\xi}-\bar{\rho} \boldsymbol{\xi} \cdot \mathbf{a}\right. \\
& \left.\quad+(\boldsymbol{\xi} \cdot \nabla) \frac{B^{2}}{2 \mu_{0}}+2 i \omega \eta \hat{\mathbf{n}} \cdot(\nabla \boldsymbol{\xi}) \cdot \hat{\mathbf{n}}\right\rangle=0 .
\end{aligned}
$$

In its full form, the equation of motion for the perturbations (4) requires the solution of a boundary layer problem. However, for weak viscosity and relatively low frequency perturbations, vorticity will be slowly generated and can be neglected in a first approximation. Furthermore, one may neglect the small modifications introduced by both the viscous forces and the acceleration on the energy required to compress the fluid, reducing the equation of motion to the standard MHD form

$$
-\rho \omega^{2} \boldsymbol{\xi}-\frac{\mathbf{B}}{\mu_{0}} \cdot \nabla \delta \mathbf{B} \cong-\nabla \delta p^{*}+\delta \mathbf{B} \cdot \frac{\nabla \mathbf{B}}{\mu_{0}} .
$$

Of course, the solution of the equation of motion in this form cannot satisfy the tangential conditions of continuity of the fluid velocity and stress across the boundary layer. Nevertheless, for free-boundary perturbations the most important contributions of the acceleration and viscosity comes from the normal pressure balance condition

$$
\begin{aligned}
& \left\langle\delta p^{*}+\boldsymbol{\xi} \cdot \nabla p-\bar{\rho} \boldsymbol{\xi} \cdot \mathbf{a}+(\boldsymbol{\xi} \cdot \nabla) \frac{B^{2}}{2 \mu_{0}}+2 i \omega \eta \hat{\mathbf{n}} \cdot(\nabla \boldsymbol{\xi}) \cdot \hat{\mathbf{n}}\right\rangle \\
& \quad \cong 0
\end{aligned}
$$

where the small contributions in the compressive energy introduced both by the acceleration and the viscous forces across the boundary layer were also neglected. The important contribution of the acceleration is the push exerted by the flow of air on the perturbed boundary. In the same way, the magnetic field exerts a pinching force on the boundary. As for the viscous stresses, they introduce a significant damping of the velocity perturbations at the edge. The remaining boundary conditions correspond to the continuity of the normal velocity perturbation at the interface between the internal and external fluids,

$$
\hat{\mathbf{n}} \cdot\langle\boldsymbol{\xi}\rangle=0,
$$

and the condition of continuity of the normal component of the magnetic induction across the perturbed boundary, which, in the presence of a surface current density $\mathbf{K}$, becomes

$$
\hat{\mathbf{n}} \cdot[\delta \mathbf{B}-\nabla \times(\boldsymbol{\xi} \times \mathbf{B})]_{r_{c}}=0 .
$$

The external azimuthal magnetic field is given in terms of the total discharge current $I$ by $B_{\theta}(r)=\mu_{0} I /(2 \pi r)$. The assumption of perfect conductivity means that the current is not distributed throughout the plasma cross section but flows in a thin layer on the surface of the plasma column. The surface current density is $K=I /\left(2 \pi r_{c}\right)$ and the magnetic field vanishes inside the discharge. Last, the fluid perturbations that retain cylindrical symmetry are of the form:

$$
\boldsymbol{\xi}=\left[\xi_{r}(r) \hat{\mathbf{r}}+\xi_{\theta}(r) \hat{\boldsymbol{\theta}}+\xi_{z}(r) \hat{\mathbf{z}}\right] \exp (i m \theta+i k z-i \omega t) .
$$

The air outside the discharge is nonconductive and can be assumed inviscid for the ambient temperature. With approximately uniform values of the gas density $\rho_{e}$ and pressure $p_{e}$, the normal mode equation for the external fluid perturbation is $\left(\gamma_{e}=7 / 5\right.$ for air in normal conditions)

$$
-\rho_{e} \omega^{2} \boldsymbol{\xi}_{e}=\gamma_{e} p_{e} \nabla\left(\nabla \cdot \boldsymbol{\xi}_{e}\right),
$$

from which, solving in terms of $\xi_{e, z}$,

$$
\xi_{e, r}=\frac{1}{i k} \frac{d \xi_{e, z}}{d r}, \quad \xi_{e, \theta}=\frac{m}{k r} \xi_{e, z}
$$

where $\xi_{e, z}$ is given by a solution of the Bessel's equation

$$
\frac{1}{r} \frac{d}{d r}\left(r \frac{d \xi_{e, z}}{d r}\right)-\left(k^{2}-\frac{\omega^{2}}{c_{e}^{2}}+\frac{m^{2}}{r^{2}}\right) \xi_{e, z}=0,
$$

and $c_{e}=\sqrt{\gamma_{e} p_{e} / \rho_{e}}$ is the speed of sound outside the plasma column. The regular solution outside the discharge $\left(r_{c}<r\right.$ $<\infty)$ is given in terms of the modified Bessel functions of the second kind

$$
\xi_{e, z}(r)=\frac{K_{m}\left(\kappa_{e} r\right)}{K_{m}\left(\kappa_{e} r_{c}\right)} \xi_{e, z}\left(r_{c}\right),
$$

where $\kappa_{e}=\sqrt{k^{2}-\omega^{2} / c_{e}^{2}}$.

Similarly, the normal mode equation for the internal fluid perturbation with approximately uniform values of the gas density $\rho_{i}$ and pressure $p_{i}$ is

$$
-\rho_{i} \omega^{2} \boldsymbol{\xi}_{i}=\gamma_{i} p_{i} \nabla\left(\nabla \cdot \boldsymbol{\xi}_{i}\right),
$$

and the regular solution inside the discharge $\left(0<r<r_{c}\right)$ is given in terms of the modified Bessel's function of the first kind 


$$
\xi_{i, z}(r)=\frac{I_{m}\left(\kappa_{i} r\right)}{I_{m}\left(\kappa_{i} r_{c}\right)} \xi_{i, z}\left(r_{c}\right),
$$

where $\kappa_{i}=\sqrt{k^{2}-\omega^{2} / c_{i}^{2}}$.

Likewise, for perturbations of the form $\phi_{m}$ $=\phi_{m}(r) \exp (i m \theta+i k z-i \omega t)$ the external magnetic potential $\phi_{m}(r)$ satisfies the Bessel's equation

$$
\frac{1}{r} \frac{d}{d r}\left(r \frac{d \phi_{m}}{d r}\right)-\left(k^{2}+\frac{m^{2}}{r^{2}}\right) \phi_{m}=0
$$

with a regular solution for $r_{c}<r<\infty$ given by

$$
\phi_{m}(r)=\frac{K_{m}(k r)}{K_{m}\left(k r_{c}\right)} \phi_{m}\left(r_{c}\right) \text {. }
$$

Now, the fluid continuity condition (13) yields a relation between $\xi_{i, z}\left(r_{c}\right)$ and $\xi_{e, z}\left(r_{c}\right)$

$$
\frac{\xi_{i, z}\left(r_{c}\right)}{\xi_{e, z}\left(r_{c}\right)}=\frac{\kappa_{e} I_{m}\left(\kappa_{i} r_{c}\right) K_{m}^{\prime}\left(\kappa_{e} r_{c}\right)}{\kappa_{i} I_{m}^{\prime}\left(\kappa_{i} r_{c}\right) K_{m}\left(\kappa_{e} r_{c}\right)},
$$

and the magnetic field continuity condition (14) gives a relation between $\xi_{e, z}\left(r_{c}\right)$ and $\phi_{m}\left(r_{c}\right)$

$$
\frac{k K_{m}^{\prime}\left(k r_{c}\right)}{K_{m}\left(k r_{c}\right)} \phi_{m}\left(r_{c}\right)+\frac{m}{k r_{c}} \frac{\kappa_{e} K_{m}^{\prime}\left(\kappa_{e} r_{c}\right)}{K_{m}\left(\kappa_{e} r_{c}\right)} \frac{B_{\theta}\left(r_{c}\right)}{\mu_{0}} \xi_{e, z}\left(r_{c}\right)=0 .
$$

Viscosity effects are relevant only inside the discharge because of the high temperature and the transient turbulent fluctuations. Therefore, the normal pressure balance condition (12) can be written in the form

$$
\begin{aligned}
& -\gamma_{e} p_{e}\left(\nabla \cdot \xi_{e}\right)_{r_{c}}+\frac{B_{\theta}\left(r_{c}\right)}{\mu_{0}}\left[\delta \mathbf{B}+\left(\xi_{e} \cdot \nabla\right) \mathbf{B}\right]_{r_{c}} \cdot \hat{\theta}+\bar{\rho} a \xi_{e}\left(r_{c}\right) \\
& \cdot \hat{\mathbf{r}}+\gamma_{i} p_{i}\left(\nabla \cdot \xi_{i}\right)_{r_{c}}+\bar{\rho} a \xi_{i}\left(r_{c}\right) \cdot \hat{\mathbf{r}}-2 i \omega \eta_{i} \hat{\mathbf{r}} \cdot\left(\nabla \xi_{i}\right)_{r_{c}} \cdot \hat{\mathbf{r}} \cong 0,
\end{aligned}
$$

Note that the acceleration vector $\mathbf{a}=a \hat{\mathbf{r}}$ is directed along the normal $\hat{\mathbf{n}}$ in the inside region and oppositely directed to the normal in the outside region. Introducing the normal mode solutions in Eq. (26) leads to a further relation between $\xi_{i, z}\left(r_{c}\right), \xi_{e, z}\left(r_{c}\right)$ and $\phi_{m}\left(r_{c}\right)$

$$
\begin{aligned}
& {\left[\frac{\rho_{e} \omega^{2}}{k}+\left(\frac{\bar{\rho} a}{k}-\frac{B_{\theta}^{2}\left(r_{c}\right)}{\mu_{0} k r_{c}}\right) \frac{\kappa_{e} K_{m}^{\prime}\left(\kappa_{e} r_{c}\right)}{K_{m}\left(\kappa_{e} r_{c}\right)}\right] \xi_{e, z}\left(r_{c}\right)} \\
& +\left(-\frac{\rho_{i} \omega^{2}}{k}+\frac{\bar{\rho} a}{k} \frac{\kappa_{i} I_{m}^{\prime}\left(\kappa_{i} r_{c}\right)}{I_{m}\left(\kappa_{i} r_{c}\right)}-\frac{2 i \omega \eta_{i}}{k} \frac{\kappa_{i}^{2} I_{m}^{\prime \prime}\left(\kappa_{i} r_{c}\right)}{I_{m}\left(\kappa_{i} r_{c}\right)}\right) \xi_{i, z}\left(r_{c}\right) \\
& +\frac{m}{r_{c}} B_{\theta}\left(r_{c}\right) \phi_{m}\left(r_{c}\right) \cong 0,
\end{aligned}
$$

which combined with Eqs. (24) and (25) gives the dispersion equation that relates $\omega, m$, and $k$ :

$$
\begin{gathered}
\left(\frac{\rho_{i} I_{m}\left(\kappa_{i} r_{c}\right)}{\kappa_{i} I_{m}^{\prime}\left(\kappa_{i} r_{c}\right)}-\frac{\rho_{e} K_{m}\left(\kappa_{e} r_{c}\right)}{\kappa_{e} K_{m}^{\prime}\left(\kappa_{e} r_{c}\right)}\right) \omega^{2}+2 \eta_{i}\left(\frac{\kappa_{i} I_{m}^{\prime \prime}\left(\kappa_{i} r_{c}\right)}{I_{m}^{\prime}\left(\kappa_{i} r_{c}\right)}\right) i \omega \\
+\left(1+m^{2} \frac{K_{m}\left(k r_{c}\right)}{k r_{c} K_{m}^{\prime}\left(k r_{c}\right)}\right) \frac{\bar{\rho} v_{A}^{2}}{r_{c}}-2 \bar{\rho} a=0,
\end{gathered}
$$

where $v_{A}=\sqrt{B_{\theta}^{2}\left(r_{c}\right) / \mu_{0} \bar{\rho}}$ is the Alfvén speed at the edge of the discharge.
For short wavelengths $\left(k r_{c} \gg 1\right)$ the dispersion relation (28) becomes independent of the azimuthal wave number $m$ :

$$
\left(\frac{\rho_{e}+\rho_{i}}{2 \bar{\rho}}\right) \omega^{2}+\frac{\eta_{i} k^{2}}{\bar{\rho}} i \omega+\left(\frac{v_{A}^{2}}{2 r_{c}}-a\right) k \cong 0,
$$

showing the strong damping effect of the viscosity in the short wavelength limit. Neglecting magnetic field and viscosity effects, the fluid perturbations in an expanding column oscillate with the real frequency $\omega \cong \sqrt{\left[2 \bar{\rho} /\left(\rho_{e}+\rho_{i}\right)\right] a k}$ typical of gravity waves. Including the magnetic field contribution, the strong initial expansion must satisfy the condition $a>v_{A}^{2} /\left(2 r_{c}\right)$ in order to overcome the pinch effect. Now, the case of interest in the present article corresponds to a contracting discharge, that is, when $a<0$. In this case the dispersion relation with negligible magnetic field in the inviscid limit describes the Rayleigh-Taylor instability at the interface between two fluids, giving a growth rate $-i \omega$ $\cong \sqrt{\left[2 \bar{\rho} /\left(\rho_{e}+\rho_{i}\right)\right]|a| k}$. According to the equilibrium condition (2), when $a<0$ and $I$ is negligible, the internal pressure is larger than the external pressure and the situation is favorable to the instability $\left(p_{i}>p_{e}, \rho_{i}<\rho_{e}\right)$. Including both the magnetic field and viscosity effects, the maximum growth rate and corresponding wave number in the short wavelength limit are calculated from Eq. (29):

$$
\begin{aligned}
& -i \omega_{\max } \cong\left[\frac{\bar{\rho}^{2}}{2\left(\rho_{e}+\rho_{i}\right) \eta_{i}}\left(\frac{v_{A}^{2}}{2 r_{c}}+|a|\right)^{2}\right]^{1 / 3}, \\
& k_{\max } \cong\left[\frac{\bar{\rho}\left(\rho_{e}+\rho_{i}\right)}{4 \eta_{i}^{2}}\left(\frac{v_{A}^{2}}{2 r_{c}}+|a|\right)\right]^{1 / 3},
\end{aligned}
$$

giving $\eta_{i} k_{\max }^{2} \cong(-i \omega)_{\max }\left(\rho_{e}+\rho_{i}\right) / 2$, which corresponds to viscosity and inertial terms of the same amplitude in the fluid equations. The maximum growth rate of the instability occurs for inverse wave numbers comparable with the scale length $L_{\rho}=\rho\left(r_{c}\right) /\left|\rho^{\prime}\left(r_{c}\right)\right| \ll r_{c}$ of the density gradient at the edge of the channel, which is estimated from the mass flow across the viscous boundary by Eq. (C29).

It will be shown in the next section that for short and intermediate wavelengths $\left(k r_{c} \gtrsim 1\right)$ there is only a small difference in the growth rates of the $m=0$ and $m=1$ modes and that Eq. (29) gives a reasonable approximation of the $m=0$ mode even for long wavelengths. Actually, the growth rate of the $m=0$ mode vanishes in the long wavelength limit $\left(k r_{c}\right.$ $\ll 1)$. However, the dispersion relation of the $m=1$ mode for long wavelengths is given approximately by

$$
\begin{gathered}
{\left[\frac{\rho_{e}}{\bar{\rho}}\left(1-\left(\log \frac{2}{\kappa_{e} r_{c}}-\gamma_{E}\right) \kappa_{e}^{2} r_{c}^{2}\right)+\frac{\rho_{i}}{\bar{\rho}}\left(1-\frac{\kappa_{i}^{2} r_{c}^{2}}{4}\right)\right] \omega^{2}} \\
\quad+\frac{3}{2} \frac{\eta_{i}}{\bar{\rho} c_{i}} \kappa_{i}^{2} i \omega+\left(\log \frac{2}{k r_{c}}-\gamma_{E}\right) k^{2} v_{A}^{2}+\frac{2|a|}{r_{c}}=0
\end{gathered}
$$

where $\gamma_{E} \cong 0.577216$ is Euler's constant. Expanding to lowest order in the growth rate and wave number yields 


$$
\begin{aligned}
-i \omega \cong & \left(\frac{2 \bar{\rho}|a|}{\left(\rho_{e}+\rho_{i}\right) r_{c}}\right)^{1 / 2}+\left(\frac{2 \bar{\rho}|a|}{\left(\rho_{e}+\rho_{i}\right) r_{c}}\right)^{1 / 2}\left[\frac { 1 } { 2 } \left(\frac{\rho_{e}}{\rho_{e}+\rho_{i}}\right.\right. \\
& \left.+\frac{v_{A}^{2}}{2|a| r_{c}}\right)\left(\log \frac{2}{k r_{c}}-\gamma_{E}\right)+\frac{\rho_{i}}{8\left(\rho_{e}+\rho_{i}\right)} \\
& \left.-\left(\frac{\bar{\rho} r_{c}}{2\left(\rho_{e}+\rho_{i}\right)|a|}\right)^{1 / 2} \frac{3 \eta_{i}}{4 \bar{\rho}_{c}^{2}}\right] k^{2} r_{c}^{2} .
\end{aligned}
$$

This result indicates that very long wavelength kink perturbations have an approximately fixed growth rate corresponding to the $e$-folding time

$$
\tau_{\infty} \sim\left(\frac{\left(\rho_{e}+\rho_{i}\right) r_{c}}{2 \bar{\rho}|a|}\right)^{1 / 2} .
$$

At the opposite limit, very short wavelength perturbations starting at $k \sim 1 / L_{\rho}$ have much larger growth rates. These perturbations introduce velocity fluctuations $\Delta u$ on a scale length $\ell$ that result in a turbulent state inside the discharge. To account for the anomalous character of the viscosity induced by these fluctuations, the dispersion relation is modified multiplying the viscosity by the Reynolds number Re $=\Delta u \ell / \nu_{i}$, where $\nu_{i}=\eta_{i} / \rho_{i}$ is the kinematic viscosity $(\operatorname{Re}=1$ corresponds to velocity fluctuations in the molecular scale). In the turbulent state the Reynolds number is estimated taking $\Delta u=K_{1} c_{i}$ and $\ell=K_{2} r_{c}$, where $K_{1}$ and $K_{2}$ are numerical coefficients. Initially, $\ell \cong L_{\rho}$ implies $K_{2} \cong L_{\rho} / r_{c} \ll 1$. For fully developed turbulence $K_{1}$ is of the order of unity and $L_{\rho} / r_{c}$ $\ll K_{2} \lesssim 1$. For air at atmospheric pressure and at $10000 \mathrm{~K}$ the kinematic viscosity is $\nu_{i} \cong 0.0060 \mathrm{~m}^{2} / \mathrm{s}$ and the sound speed is $c_{i} \cong 2.0 \mathrm{~km} / \mathrm{s}$. Therefore, values of the Reynolds number up to $\mathrm{Re} \sim 10000$ can be expected in the turbulent state of a lightning discharge with a current channel radius $r_{c}$ of the order of a few centimeters, which is the radius at the final decay stages of the return stroke current according to most experiments and models, and consistently with the calculations presented in Sec. III. Introducing the Reynolds number in Eq. (30) results in the following scalings for the $e$-folding time and the wavelength of the instability at maximum growth rate:

$$
\begin{aligned}
\tau_{\max } & \cong\left[\frac{2\left(\rho_{e}+\rho_{i}\right) \eta_{i}}{\bar{\rho}^{2}}\left(\frac{v_{A}^{2}}{2 r_{c}}+|a|\right)^{-2}\right]^{1 / 3} \mathrm{Re}^{1 / 3}, \\
\lambda_{\max } & \cong 2 \pi\left[\frac{4 \eta_{i}^{2}}{\bar{\rho}\left(\rho_{e}+\rho_{i}\right)}\left(\frac{v_{A}^{2}}{2 r_{c}}+|a|\right)^{-1}\right]^{1 / 3} \mathrm{Re}^{2 / 3} .
\end{aligned}
$$

As pointed out previously, these scalings give reasonable results for the $m=0$ sausage mode at all wavelengths, and for the $m=1$ kink mode at short wavelengths. In the long wavelength limit, Eq. (32) gives the wavelength of maximum growth rate of the $m=1$ mode:

$$
\begin{aligned}
\lambda_{\max } \cong & \pi r_{c} \exp \left\{\gamma_{E}+\frac{1}{2}+\frac{\rho_{i}|a| / 4}{\rho_{e}|a|+\left(\rho_{e}+\rho_{i}\right) v_{A}^{2} /\left(2 r_{c}\right)}\right. \\
& \left.\times\left[\left(\frac{\left(\rho_{e}+\rho_{i}\right) r_{c}}{2 \bar{\rho}|a|}\right)^{1 / 2} \frac{6 \eta_{i}}{\rho_{i} r_{c}^{2}} \operatorname{Re}-1\right]\right\} .
\end{aligned}
$$

This formula gives a crude approximation of the wavelength corresponding to maximum growth rate of the kink mode for very large values of Re (fully developed turbulent state).

\section{RESULTS}

In this section the results of the analysis carried out in Sec. II are applied to a lightning discharge of peak current $I_{0}=45 \mathrm{kA}$ as shown in Figs. 1 and 2. Standard atmospheric parameters are used, namely, $M_{0}=28.964 \mathrm{~kg} / \mathrm{mol}, p_{e}=p_{0}$ $=1.01325 \times 10^{5} \mathrm{~Pa}, \quad \rho_{e}=\rho_{0}=1.22496 \mathrm{~kg} / \mathrm{m}^{3}, \quad c_{e}=c_{0}$ $=340.29 \mathrm{~m} / \mathrm{s}$, and $\eta_{0}=1.7894 \times 10^{-5} \mathrm{~Pa} \cdot \mathrm{s}$. The energy input at current peak is estimated using Eq. (B6):

$$
\mathcal{E}_{0}=1.69\left(\frac{\rho_{0} I_{0}^{4}}{\left[4 \pi \sigma_{0} C^{2}(\gamma) \zeta_{c}^{2} / 3\right]^{2}}\right)^{1 / 3}=3.30 \mathrm{~kJ} / \mathrm{m},
$$

where $C(7 / 5)=1.00402$ is the parameter that conserves the excess energy in a strong cylindrical shock wave in air (cf. Appendix A), $\sigma_{0}=2.225 \times 10^{4} \mathrm{~S} / \mathrm{m}$ is the value assumed by Braginskii ${ }^{16}$ for the conductivity of the current channel, and $\zeta_{c}=0.526$ is the constant that defines the position of the current channel behind the shock wave, in accordance with the model presented in the Appendix B. The energy $\mathcal{E}_{0}$ is the input that drives the shock wave associated with the lightning discharge, taking roughly into account the energy lost by radiation for a total energy input given by Eq. (B7). The position where the excess energy $\mathcal{E}_{0}$ equals the background thermal energy is the characteristic radius $R_{0}$ $=\sqrt{(\gamma-1) \mathcal{E}_{0} /\left(\pi p_{0}\right)} \cong 6.44 \mathrm{~cm}$ defined by Eq. (A19), and the characteristic time is $\tau_{0}=R_{0} / c_{0} \cong 189 \mu \mathrm{s}$. Pressure equilibration on axis occurs at $t_{\mathrm{eq}}=0.260 \tau_{0} \cong 49 \mu \mathrm{s}$ and the onset of air backflow at the current channel occurs at $t_{b}=0.53 \tau_{0}$ $\cong 100 \mu \mathrm{s}$, in accordance with the estimates presented in the Appendices A and C, respectively. Using Eqs. (C1) and (C2), the peak velocity of the backflow compression wave at the channel boundary is estimated by $v_{m} \cong 0.013 c_{0}=4.42 \mathrm{~m} / \mathrm{s}$ and the acceleration by

$$
a_{0} \cong 0.093\left(\gamma p_{0}\right)^{3 / 2} /\left(\rho_{0} \mathcal{E}_{0}^{1 / 2}\right)=7.07 \times 10^{4} \mathrm{~m} / \mathrm{s}^{2},
$$

respectively. The acceleration scale length is $\left(L_{a}\right)_{0}$ $\cong 0.331 R_{0}=2.13 \mathrm{~cm}$ and the equilibrium position of the current channel at the onset of air backflow is $r_{c}\left(t_{b}\right) \cong R_{0}$ $-\left(L_{a}\right)_{0}=4.31 \mathrm{~cm}$, estimated by Eqs. (C24) and (C25), respectively.

The temperature on the edge of the channel is assumed equal to the temperature at pressure equilibration on axis, which is $T_{\text {eq }} \cong 3800 \mathrm{~K}$ in accordance with Eq. (B10). At this temperature the dynamic viscosity of air is $\eta_{i} \cong 1.12$ $\times 10^{-4} \mathrm{~kg} /(\mathrm{m} \mathrm{s})$, the kinematic viscosity is $\nu_{i}$ $\cong 0.0012 \mathrm{~m}^{2} / \mathrm{s}$ for $\rho_{i} \cong 9.46 \times 10^{-2} \mathrm{~kg} / \mathrm{m}^{3}$, and the sound speed is $c_{i} \cong 1.24 \mathrm{~km} / \mathrm{s}$. During the backflow stage the overpressure at the channel boundary is very small, being estimated by Eq. (C9) as $\Delta p / p_{0} \cong \gamma\left(v_{m} / c_{0}\right)=0.018$. Also, the average density $\bar{\rho}$ in the backflow compression wave is approximately equal to the external density $\rho_{e}=\rho_{0}$. For approximately constant pressure the mass flow across the viscous boundary determines the scale length of the density distribution, which is calculated by Eq. (C29) as $L_{\rho}$ $\cong 16 \eta_{i} /\left(3 \rho_{0} v_{m}\right)=0.11 \mathrm{~mm}$ at a temperature of $3800 \mathrm{~K}$. 

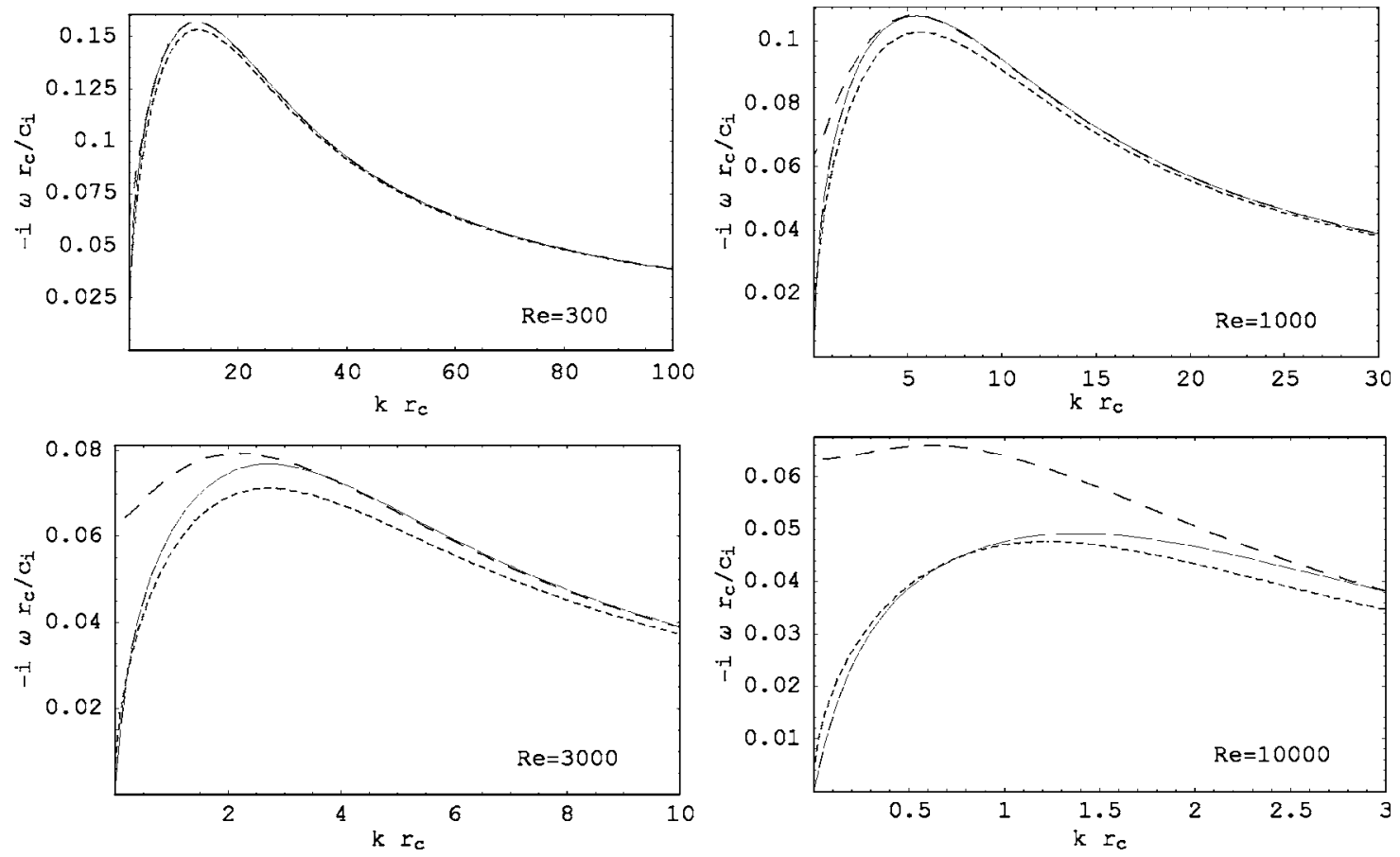

FIG. 3. Normalized growth rate of the instabilities in a lightning discharge as a function of the normalized wave number for various values of the Reynolds number. The continuous line corresponds to the $m=0$ sausage instability and the dashed line to the $m=1$ kink instability. The short dashed line corresponds to the short wavelength asymptotic limit.

At the onset of air backflow $\left(t_{b} \cong 100 \mu \mathrm{s}\right)$, Fig. 2 shows that the current in the discharge decayed to values below 10 kA. Assuming $I \leqslant 10 \mathrm{kA}$, the effective acceleration associated with the magnetic pinching forces is

$$
\frac{v_{A}^{2}}{2 r_{c}\left(t_{b}\right)}=\frac{\mu_{0} I^{2}}{8 \pi^{2} \bar{\rho} r_{c}^{3}\left(t_{b}\right)} \leqslant 1.62 \times 10^{4} \mathrm{~m} / \mathrm{s}^{2},
$$

which is significantly less than the air backflow acceleration $a_{0} \cong 7.07 \times 10^{4} \mathrm{~m} / \mathrm{s}^{2}$. Therefore, during most of the continuing current stage, with a typical current $I_{c}=1000-100 \mathrm{~A},{ }^{5}$ the pinch instability is much weaker then the RayleighTaylor instability. In the following calculations, the continuing current is assumed constant, with a value $I_{c}=1000 \mathrm{~A}$.

Figure 3 shows the normalized growth rate, $-i \omega r_{c} / c_{i}$, versus the normalized wave number, $k r_{c}$, of the instabilities in a lightning discharge including anomalous viscosity effects. The continuous line corresponds to the $m=0$ sausage instability, the dashed line to the $m=1$ kink instability, and the short dashed line to the short wavelength limit. The plots correspond to steps in the course of air backflow in a discharge with a peak current $I_{0}=45 \mathrm{kA}$ and a temperature at the edge of the channel $T_{\text {eq }} \cong 3800 \mathrm{~K}$. The exact value of this temperature does not significantly affect the results. The Reynolds number takes the values $\mathrm{Re}=300,1000,3000$, and 10000 , as indicated in Fig. 3. The plots clearly show the shift of the maximum growth rate to larger wavelengths when the Reynolds number increases. Also, they show that the short wavelength limit corresponding to Eq. (29) gives a reasonable approximation of the $m=0$ mode at all wavelengths. The growth rate of the $m=0$ mode vanishes for $\lambda$ $\rightarrow \infty$, but the limit $e$-folding time of the $m=1$ mode is $\tau_{\infty}$ $\cong 0.551 \mathrm{~ms}$, which is weakly dependent on the value of the viscosity and can be estimated by Eq. (33). This corresponds to the reaction against the compressive force piling up air inside the channel.

Table I gives the maximum growth rates and corresponding wave numbers of the sausage and kink instabilities in a $45 \mathrm{kA}$ discharge for $\mathrm{Re}=1$ (molecular viscosity) and $\mathrm{Re}$ $\sim 10000$ (fully developed turbulence). At the classical level of the viscosity there is no difference between the growth rates and wave numbers for both instabilities. However, for the largest scale velocity fluctuations, the characteristic wavelength of the kink instability is $\sim 0.44 \mathrm{~m}$, about twice the wavelength of the sausage instability. These results are in rough agreement with the structures that can be inferred from Fig. 1. Finally, Fig. 4 shows the wavelength of maximum growth rate of the $m=1$ mode as a function of the peak return current in the range from 15 to $120 \mathrm{kA}$ at the final stages of the instability. The thin continuous line corresponds to Re $=10000$, the long dashed line to $\operatorname{Re}=7000$, and the short dashed line to $R e=5000$. One verifies that for values of the Reynolds number near $\mathrm{Re}=10000$ the $0.5 \mathrm{~m}$ wavelength becomes a characteristic of all discharges. It is practically in-

TABLE I. Maximum growth rates (e-folding times) and corresponding wave numbers (wavelengths) of the $m=0$ (sausage) and the $m=1$ (kink) instabilities in a $45 \mathrm{kA}$ lightning discharge for extreme values of the Reynolds number.

\begin{tabular}{ccc}
\hline \hline Reynolds number & $-i \omega_{\max } r_{c} / c_{i}$ & $\left(k r_{c}\right)_{\max }$ \\
\hline $1(m=0,1)$ & $1.026(34.0 \mu \mathrm{s})$ & $567(0.477 \mathrm{~mm})$ \\
$10000(m=0)$ & $0.0492(0.708 \mathrm{~ms})$ & $1.39(19.5 \mathrm{~cm})$ \\
$10000(m=1)$ & $0.0659(0.529 \mathrm{~ms})$ & $0.618(43.8 \mathrm{~cm})$ \\
\hline \hline
\end{tabular}




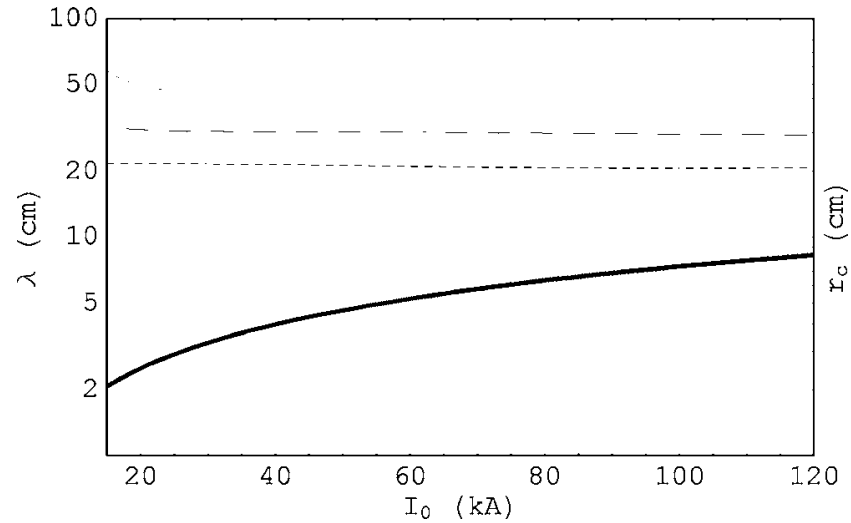

FIG. 4. Characteristic wavelength of the kink instability and channel radius of a lightning discharge as a function of the peak return current. The thick continuous line shows the channel radius, at the onset of air backflow, ranging from 2.1 to $8.3 \mathrm{~cm}$. The thin continuous line, the long dashed line and the short dashed line show the characteristic wavelength corresponding to $\operatorname{Re}=10000,7000$, and 5000, respectively.

dependent of the current with a rather larger value in the low current range. However, one would expect that the final level of turbulence depends on the energy input, giving a smaller value of the characteristic wavelength for smaller values of the peak current in the lightning flash. The estimated channel radius at quasiequilibrium is also shown in Fig. 4, varying proportionally to $I_{0}^{2 / 3}$ in the approximate range from 2.1 to $8.3 \mathrm{~cm}$. The curves shown in Fig. 4 are calculated from the exact dispersion equation, whereas Eq. (35) gives only a crude approximation.

\section{CONCLUSIONS}

The following picture of the discharge evolution emerges. At early times in the backflow stage, the instabilities with a large growth rate have an inverse wave number of the order of the density scale length $L_{\rho}$, which is defined by the classical viscosity and is very short compared with the channel radius $r_{c}$. Then, during the course of air backflow, the turbulence develops and the viscosity becomes anomalous, shifting the wavelength of the most unstable modes to values of the order of and larger than the channel radius $r_{c}$. The large scale fluctuations fill the arc channel in a few milliseconds. In the final state of turbulence the most unstable kink mode has a characteristic wavelength that can be as large as $0.5 \mathrm{~m}$, with a relatively small growth rate during the persistence of the backflow process. The whole development takes possibly $5 \mathrm{~ms}$, according to Fig. 1, when the RayleighTaylor instability quenches and the spatial structure becomes frozen, except for the very small growth rate of the pinch instability associated with the continuing current. Also, enhanced diffusion is expected while the instability lasts and before the turbulence decays in the absence of a driving energy source. The beaded configuration corresponds to a diffuse channel showing the frozen spatial structure of the instabilities during the history of their evolution. After that, sections of large radius take longer to cool than channels of small radius, having a longer lifetime according to the model proposed by Uman and Voshall ${ }^{9}$ and the calculations carried out by Boichenko. ${ }^{10}$
Note that the requirements on the final level of anomalous viscosity can be relaxed in two ways. First, an examination of Eqs. (34) and (35) shows that the wavelength of maximum instability increases with the decrease of the acceleration. So, in the final stages of the Rayleigh-Taylor instability there is an enhancement of the long wavelength distortions, although with a diminishing growth rate. Further, the region of hydrodynamic instability moves outwards during the air backflow stage, as pointed out in the Sec. I and by Hill. ${ }^{14}$ This means that the reference radius in Eq. (35), for example, is on average larger than the current channel radius $r_{c}$, if one considers only the hydrodynamic contribution.

It is interesting to point out that for very large axial wavelengths, the kink mode is weakly unstable with an $e$-folding time given approximately by Eq. (33). At the peak value of the acceleration, a $5 \mathrm{~ms}$ period would allow about nine exponentiations, causing appreciable deformation of any initial perturbation. Assuming an initial small deformation of the lightning channel caused by periodic structures in the neutral atmosphere, like internal gravity waves, this could explain the occurrence of natural bead lightning with luminous fragments of a few meters to some tens of meters in length, as reported in the book by Rakov and Uman. ${ }^{6}$ Finally, the persistency of the pinch instability during the continuing current stage and its strengthening in a M-component event could, as mentioned in Sec. I, enhance the deformations in the $10 \mathrm{~cm}$ wavelength range, a phenomenon observed by Idone. ${ }^{17}$

Of course, further work is needed to obtain a precise picture of the processes involved in the formation of bead lightning. Some of the topics that need to be addressed more carefully are the actual level of turbulence reached in the discharge and the total duration and intensity of air backflow, problems suitable to be investigated by simulation. Nevertheless, it is remarkable that, even in the overly simplified form presented in this article, the linear instability theory gives hints to this highly dynamic and nonlinear process.

\section{ACKNOWLEDGMENT}

One of the authors (G.O.L.) acknowledges partial support for this work from the International Atomic Energy Agency under Research Contract No. BRA-12932.

\section{APPENDIX A: CYLINDRICAL SHOCK WAVES}

The theory of cylindrical shock waves is usually applied to the study of lightning discharges, ${ }^{18}$ resulting in the simplest model of the phenomenon. Theoretical development on this subject started with the similarity solution of spherical shock waves introduced by Sedov ${ }^{19,20}$ and Taylor, ${ }^{21}$ in relation to the study of atomic explosions. The similarity solution was extended in the early 1950 s to cylindrical blast waves $^{22-24}$ and the theoretical predictions were later compared with the results of laboratory experiments using an inverse pinch machine. ${ }^{25}$ The analytic solution for a strong cylindrical shock wave is here briefly reviewed to introduce the expressions used for estimating the parameters in the return stroke of a lightning discharge. 
The ideal fluid motion in cylindrical coordinates is governed by the continuity, momentum and energy equations, given respectively by

$$
\begin{aligned}
& \frac{\partial \rho}{\partial t}+\frac{\partial}{\partial r}(\rho v)+\frac{\rho v}{r}=0, \\
& \rho\left(\frac{\partial v}{\partial t}+v \frac{\partial v}{\partial r}\right)+\frac{\partial p}{\partial r}=0, \\
& \rho\left(\frac{\partial p}{\partial t}+v \frac{\partial p}{\partial r}\right)-\gamma p\left(\frac{\partial \rho}{\partial t}+v \frac{\partial \rho}{\partial r}\right)=0,
\end{aligned}
$$

where $\rho$ is the mass density, $v$ is the velocity of motion of the gas in the rest frame, $p$ is the pressure, and $\gamma$ is the ratio of specific heats. The jump (Rankine-Hugoniot) conditions at the shock are

$$
\langle\rho v\rangle=0, \quad\left\langle p+\rho v^{2}\right\rangle=0, \quad\left\langle\frac{v^{2}}{2}+h\right\rangle=0,
$$

where $h=e+p / \rho$ is the specific enthalpy, which becomes $h$ $=\gamma p /[(\gamma-1) \rho]$ for a perfect gas, and $e$ is the specific internal energy. The jump conditions give the boundary conditions at $r_{0}$ immediately behind the shock wave:

$$
\begin{aligned}
\frac{\rho\left(r_{0}\right)}{\rho_{0}} & =\frac{(\gamma+1) M^{2}}{(\gamma-1) M^{2}+2}, \\
\frac{p\left(r_{0}\right)}{p_{0}} & =\frac{2 \gamma M^{2}-(\gamma-1)}{\gamma+1}, \\
\frac{v\left(r_{0}\right)}{c_{0}} & =\frac{2\left(M^{2}-1\right)}{(\gamma+1) M},
\end{aligned}
$$

where $\rho_{0}, p_{0}$, and $c_{0}$ refer to the conditions in front of the shock. The Mach number is $M=u / c_{0}$, where $u=d r_{0} / d t$ denotes the shock velocity in the rest frame, $c_{0}=\sqrt{\gamma p_{0} / \rho_{0}}$ is the sound speed in front of the shock and the gas is assumed initially to be at rest. Now, in the expression $v^{2} / 2+h$ of the fluid energy per unit mass the specific enthalpy $h$ includes the expansion work done by the gas on its surrounding. At constant volume the specific energy is $v^{2} / 2+e$ and the total excess energy per unit length released at $t=0$ is given by

$$
\begin{aligned}
\mathcal{E} & =2 \pi \int_{0}^{r_{0}}\left[\rho\left(\frac{v^{2}}{2}+e\right)-\rho_{0} e_{0}\right] r d r \\
& =2 \pi \int_{0}^{r_{0}}\left(\rho \frac{v^{2}}{2}+\frac{p}{\gamma-1}-\frac{p_{0}}{\gamma-1}\right) r d r,
\end{aligned}
$$

which remains constant for all times.

The system of equations (A1) has an integral of motion for the shock wave problem. Following the development of Landau for the spherical case, ${ }^{26}$ the energy flowing through the cylindrical surface of radius $r$ during the time $d t$ is $d t 2 \pi r \rho v\left(v^{2} / 2+h\right)$. During this time increment the volume of the cylinder expanding with the shock velocity $u$ increases by the element $d t 2 \pi r u$, which contains a gas with the excess energy density $\rho\left(v^{2} / 2+e\right)-\rho_{0} e_{0}$. Equating these two expres- sions for the energy, it follows that $\rho v\left(v^{2} / 2+h\right)=u\left[\rho\left(v^{2} / 2\right.\right.$ $\left.+e)-\rho_{0} e_{0}\right]$. For a perfect gas, this gives the integral of motion

$$
\frac{p}{\gamma-1}(\gamma v-u)+\frac{p_{0}}{\gamma-1} u=\frac{\rho v^{2}}{2}(u-v) .
$$

This relation automatically satisfies the boundary conditions (A3).

The cylindrical shock wave problem has two independent variables $(r, t)$, three dependent variables $(\rho, v, p)$, and four parameters $\left(\mathcal{E}, \rho_{0}, p_{0}, \gamma\right)$, which characterize the initial state of the gas. A self-similar solution of the problem exists if the independent variables $(r, t)$ are functions of a single variable. Introducing the similarity variable $\zeta=r / r_{0}(t)$ and the dimensionless variables $\hat{\rho}(\zeta), \hat{p}(\zeta)$, and $\hat{v}(\zeta)$ such that

$$
\begin{aligned}
& \rho(r, t)=\rho_{0} \hat{\rho}(\zeta), \\
& p(r, t)=\rho_{0}\left(\frac{d r_{0}}{d t}\right)^{2} \hat{p}(\zeta), \\
& v(r, t)=\frac{d r_{0}}{d t} \hat{v}(\zeta),
\end{aligned}
$$

the equations of motion reduce to

$$
\begin{aligned}
& \hat{\rho}\left(\frac{d \hat{v}}{d \zeta}+\frac{\hat{v}}{\zeta}\right)-(\zeta-\hat{v}) \frac{d \hat{\rho}}{d \zeta}=0, \\
& \frac{1}{r_{0}}\left(\frac{d r_{0}}{d t}\right)^{2}\left(\frac{d \hat{p}}{d \zeta}-\hat{\rho}(\zeta-\hat{v}) \frac{d \hat{v}}{d \zeta}\right)+\hat{\rho} \hat{v} \frac{d^{2} r_{0}}{d t^{2}}=0, \\
& \frac{1}{r_{0}}\left(\frac{d r_{0}}{d t}\right)^{2}(\zeta-\hat{v})\left(\gamma \hat{p} \frac{d \hat{\rho}}{d \zeta}-\hat{\rho} \frac{d \hat{p}}{d \zeta}\right)+2 \hat{p} \hat{\rho} \frac{d^{2} r_{0}}{d t^{2}}=0,
\end{aligned}
$$

with the shock location corresponding to $\zeta=1$. For $r_{0}(t)$ $=C(\gamma)\left(\mathcal{E} / \rho_{0}\right)^{1 / 4} \sqrt{t}$, where $C(\gamma)$ is a dimensionless parameter, these equations take the self-similar form:

$$
\begin{aligned}
& \frac{d \hat{v}}{d \zeta}-(\zeta-\hat{v}) \frac{d \ln \hat{\rho}}{d \zeta}+\frac{\hat{v}}{\zeta}=0, \\
& \frac{1}{\hat{\rho}} \frac{d \hat{p}}{d \zeta}-(\zeta-\hat{v}) \frac{d \hat{v}}{d \zeta}-\hat{v}=0, \\
& (\zeta-\hat{v}) \frac{d}{d \zeta} \ln \left(\frac{\hat{\rho}^{\gamma}}{\hat{p}}\right)-2=0 .
\end{aligned}
$$

The ratio $\mathcal{E} / \rho_{0}$ is the only dimensional combination containing only length and time, meaning that counterpressure effects are neglected in the self-similar solution. The parameter $C(\gamma)$ is determined from the excess energy integral (A4)

$$
\frac{4}{C^{4}(\gamma)}=2 \pi \int_{0}^{1}\left(\hat{\rho} \frac{\hat{v}^{2}}{2}+\frac{\hat{p}}{\gamma-1}\right) \zeta d \zeta-\frac{\pi}{\gamma(\gamma-1) M^{2}(t)},
$$

where $M^{2}(t)=C^{2}(\gamma)\left(\rho_{0} \mathcal{E}\right)^{1 / 2} /\left(4 \gamma p_{0} t\right)$. This shows that the similarity solution is valid for the strong shock assumption 
$M^{2}(t) \gg 1$, that is, $p_{0} t \ll\left(\rho_{0} \mathcal{E}\right)^{1 / 2}$ with $C(\gamma)$ being calculated by

$$
C(\gamma) \cong\left[\frac{\pi}{2} \int_{0}^{1}\left(\hat{\rho} \frac{\hat{v}^{2}}{2}+\frac{\hat{p}}{\gamma-1}\right) \zeta d \zeta\right]^{-1 / 4}
$$

In dimensionless variables, the integral of motion (A5) becomes

$$
\frac{\hat{p}}{\gamma-1}\left(\gamma \frac{\hat{v}}{\zeta}-1\right)+\frac{1}{\gamma(\gamma-1) M^{2}(t)}=\hat{\rho} \frac{\hat{v}^{2}}{2}\left(1-\frac{\hat{v}}{\zeta}\right)
$$

which is also valid in the self-similar form for $M^{2}(t) \gg 1$. Applied to the boundary conditions (A3) this assumption yields

$$
\begin{aligned}
& \hat{\rho}(1) \cong \frac{\gamma+1}{\gamma-1}, \\
& \hat{p}(1) \cong \frac{2}{\gamma+1}, \\
& \hat{v}(1) \cong \frac{2}{\gamma+1} .
\end{aligned}
$$

Introducing the new variable $\varphi(\zeta)=(\gamma+1) \hat{v}(\zeta) /(2 \zeta)$ so that $\varphi(1)=1$, and using the identity $d / d \zeta \equiv(d \varphi / d \zeta) d / d \varphi$, the self-similar continuity, momentum and energy equations (A8) can be written, respectively, in the form

$$
\begin{aligned}
& \frac{d \ln \hat{\rho}}{d \varphi}=\frac{2(2 \varphi+\zeta d \varphi / d \zeta)}{(\gamma+1-2 \varphi) \zeta d \varphi / d \zeta}, \\
& \frac{d}{d \varphi} \ln \left(\frac{\hat{p} / \zeta^{2}}{\hat{\rho}}\right)+\frac{d \ln \hat{\rho}}{d \varphi} \\
&= \frac{2}{\zeta d \varphi / d \zeta}+\frac{2}{\zeta d \varphi / d \zeta}\left(\frac{\hat{p} / \zeta^{2}}{\hat{\rho}}\right)^{-1} \\
& \times \frac{(\gamma+1-2 \varphi) \zeta d \varphi / d \zeta+2(\gamma+1-\varphi) \varphi}{(\gamma+1)^{2}}, \\
& \frac{d}{d \varphi} \ln \left(\frac{\hat{p} / \zeta^{2}}{\hat{\rho}}\right)-(\gamma-1) \frac{d \ln \hat{\rho}}{d \varphi}=-\frac{4(\gamma+1-\varphi)}{(\gamma+1-2 \varphi) \zeta d \varphi / d \zeta}
\end{aligned}
$$

Using the integral of motion (A11) in the form appropriate for a strong shock

$$
\frac{\hat{p} / \zeta^{2}}{\hat{\rho}} \cong \frac{2(\gamma-1)(\gamma+1-2 \varphi) \varphi^{2}}{(\gamma+1)^{2}(2 \gamma \varphi-\gamma-1)}
$$

the solution of the equations of motion is obtained in a parametric form by integration:

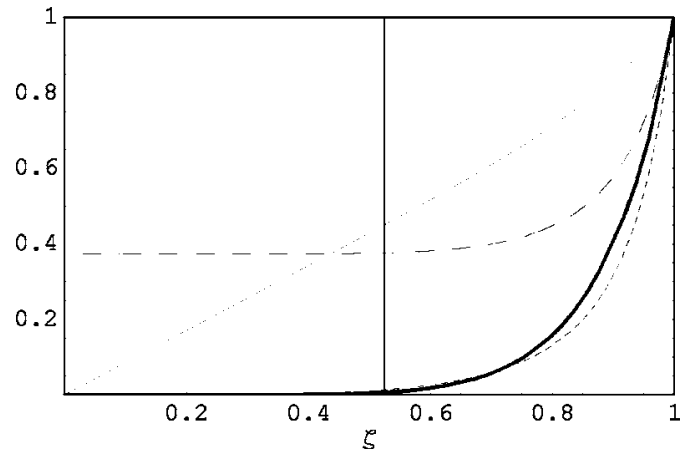

FIG. 5. Distributions of the velocity $\hat{v}(\zeta) / \hat{v}(1)$ (thin line), pressure $\hat{p}(\zeta) / \hat{p}(1)$ (dashed line) and density $\hat{\rho}(\zeta) / \hat{\rho}(1)$ (short dashed line) behind the strong shock front. The thick continuous line gives the distribution of the Spitzer resistivity $\hat{\eta}_{S}(\zeta) / \hat{\eta}_{S}(1)$. The central vertical line corresponds to the position of the current channel.

$$
\begin{aligned}
\zeta(\varphi) \cong & \frac{1}{[(\gamma+1-\gamma \varphi) \varphi]^{1 / 2}}\left(\frac{2 \gamma \varphi-\gamma-1}{\gamma-1}\right)^{(\gamma-1) /(2 \gamma)}, \\
\hat{\rho}(\varphi) \cong & \frac{\gamma+1}{\gamma-1}\left(\frac{(\gamma-1)(\gamma+1-\gamma \varphi)}{\gamma+1-2 \varphi}\right)^{2 /(2-\gamma)} \\
& \times\left(\frac{2 \gamma \varphi-\gamma-1}{\gamma-1}\right)^{1 / \gamma}, \\
\hat{p}(\varphi) \cong & \frac{2 \varphi}{\gamma+1}\left(\frac{(\gamma-1)(\gamma+1-\gamma \varphi)}{\gamma+1-2 \varphi}\right)^{\gamma /(2-\gamma)}, \\
\hat{v}(\varphi) \cong & \frac{\mathrm{A} 15)}{(\gamma+1)[(\gamma+1-\gamma \varphi) \varphi]^{1 / 2}}\left(\frac{2 \gamma \varphi-\gamma-1}{\gamma-1}\right)^{(\gamma-1) /(2 \gamma)} .
\end{aligned}
$$

The parameter $\varphi$ varies in the range $(\gamma+1) /(2 \gamma) \leqslant \varphi \leqslant 1$ for $0 \leqslant \zeta \leqslant 1$ and the solution (A15) yields the limiting values when $\zeta \rightarrow 0$ :

$$
\begin{aligned}
\hat{\rho}(\zeta) \rightarrow & 2^{-2 /[(2-\gamma)(\gamma-1)]} \gamma^{(3 \gamma-4) /[(2-\gamma)(\gamma-1)]}(\gamma-1)^{-1} \\
& \times(\gamma+1)^{(\gamma+1) /(\gamma-1)} \zeta^{2 /(\gamma-1)}, \\
\hat{p}(\zeta) \rightarrow & 2^{-\gamma /(2-\gamma)} \gamma^{2(\gamma-1) /(2-\gamma)}, \\
\hat{v}(\zeta) \rightarrow & \zeta / \gamma .
\end{aligned}
$$

Finally, substitution of the solution (A15) into Eq. (A10) gives the value of the parameter $C(\gamma)$ in quadrature form

$$
\begin{aligned}
C(\gamma) \cong & \pi(\gamma-1)^{2 /(2-\gamma)+1 / \gamma-2} \\
& \times \int_{(\gamma+1) /(2 \gamma)}^{1}\left(\frac{\gamma+1-\gamma \varphi}{\gamma+1-2 \varphi}\right)^{2 /(2-\gamma)} \\
& \left.\times \frac{2 \gamma \varphi^{2}-2(\gamma+1) \varphi+\gamma+1}{(\gamma+1-\gamma \varphi)^{3}(2 \gamma \varphi-\gamma-1)^{1 / \gamma}} d \varphi\right]^{-1 / 4} .
\end{aligned}
$$

For $\gamma=7 / 5$ this integral gives $C(7 / 5)=1.00402$. Figure 5 shows the normalized distributions of the velocity $\hat{v}(\zeta) / \hat{v}(1)$ (thin continuous line), pressure $\hat{p}(\zeta) / \hat{p}(1)$ (dashed line) and density $\hat{\rho}(\zeta) / \hat{\rho}(1)$ (short dashed line), behind the strong 
shock front. This figure also shows the distribution of the Spitzer resistivity $\hat{\eta}_{S}(\zeta) / \hat{\eta}_{S}(1)$ (thick continuous line) that will be considered in Appendix B. The distributions of the density, pressure, and velocity behind the shock wave are given by

$$
\begin{aligned}
& \rho(r, t) / \rho_{0} \cong \hat{\rho}(\zeta), \\
& p(r, t) / p_{0} \cong \gamma M^{2}(t) \hat{p}(\zeta), \\
& v(r, t) / c_{0} \cong M(t) \hat{v}(\zeta),
\end{aligned}
$$

with $\zeta=r / r_{0}(t)$. In particular, pressure equilibration occurs on axis, that is, $p\left(0, t_{\mathrm{eq}}\right)=p_{0}$, for $M^{2}\left(t_{\mathrm{eq}}\right)=1 /[\gamma \hat{p}(0)]$ $=(2 / \gamma)^{\gamma /(2-\gamma)}=2.30$, which is marginally in the strong shock regime.

The strong shock solution does not contain characteristic length and time scales. In general, a characteristic radius $R_{0}$ is defined by the position where the excess energy $\mathcal{E}$ equals the background thermal energy

$$
\mathcal{E}=2 \pi \int_{0}^{R_{0}} \frac{p_{0}}{\gamma-1} r d r=\frac{p_{0}}{\gamma-1} \pi R_{0}^{2}
$$

and the characteristic time is $\tau_{0}=R_{0} / c_{0}$. In this case, pressure equilibration occurs for $t_{\mathrm{eq}} / \tau_{0}=(1 / 4) C^{2}(\gamma)[\pi \gamma /(\gamma$ $-1)]^{1 / 2} \hat{p}(0)=0.260 \quad$ and $\quad r_{0}\left(t_{\mathrm{eq}}\right) / R_{0}=(1 / 2) C^{2}(\gamma)[\pi \hat{p}(0) /(\gamma$ $-1)]^{1 / 2}=0.787$. At this point the strong shock assumption is not strictly valid. Formally, the problem now involves a characteristic distance $R_{0}$ that turns the self-similar solution invalid.

The distributions (A18) can be compared with the numerical solutions for cylindrical shock waves obtained by Plooster, ${ }^{27}$ who used the normalized coordinates $\lambda_{P}=r / R_{P}$ and $\quad \tau_{P}=c_{0} t / R_{P}, \quad$ where $\quad R_{P}=\left[C^{4}(\gamma) \mathcal{E} /\left(4 \gamma p_{0}\right)\right]^{1 / 2}$ $=\left[\pi C^{4}(\gamma) /(4 \gamma(\gamma-1))\right]^{1 / 2} R_{0}=1.19 R_{0}$. In Plooster's coordinates, the pressure equilibration on axis calculated from the strong shock solution corresponds to $\lambda_{P}=0.660$ and $\tau_{P}$ $=0.218$. Following the initial work by Plooster, ${ }^{27-29}$ the fluid dynamic modeling of the return stroke of lightning discharges has been mostly carried out by the means of numerical simulations. ${ }^{12,30}$

\section{APPENDIX B: THE CURRENT CHANNEL}

At the point behind the strong shock wave where the pressure becomes approximately constant (see Fig. 5) the temperature increases sharply and the air conductivity increases correspondingly. During a return stroke the current that flows in this region heats the air, forming a channel with very high and approximately constant temperature. The ionization of the gas entering the channel takes place in a thin shell at the channel boundary.

At high degrees of ionization the electrical conductivity is proportional to $T^{3 / 2}$ according with Spitzer's formula. Neglecting the weak dependence of the Coulomb logarithm on the temperature, the distribution of resistivity is given by $\hat{\eta}_{S}(\zeta) / \hat{\eta}_{S}(1)=[\hat{T}(1) / \hat{T}(\zeta)]^{3 / 2}$ with $\hat{T}(\zeta)=\hat{p}(\zeta) / \hat{\rho}(\zeta)$. This distribution of the resistivity is displayed by the thick continuous line in Fig. 5, showing that the air becomes highly con- ductive for $\zeta \preceq 0.5$. Note that, as already pointed out, Spitzer's formula is strictly valid only after a relatively high degree of ionization has been reached.

The electrical energy input for a current $I(t)$ is

$$
\begin{aligned}
\mathcal{E}\left(t_{0}\right) & =\int_{0}^{t_{0}} \frac{I^{2}(t) d t}{2 \pi \int_{0}^{r_{c}} \sigma r d r} \\
& =\int_{0}^{t_{0}} \frac{I^{2}(t) d t}{2 \pi C^{2}(\gamma)\left[\mathcal{E}(t) / \rho_{0}\right]^{1 / 2} t \int_{0}^{\zeta_{c}} \sigma \zeta d \zeta} \\
& =\frac{\rho_{0}^{1 / 2}}{\pi \sigma_{0} C^{2}(\gamma) \zeta_{c}^{2}} \int_{0}^{t_{0}} \frac{I^{2}(t) d t}{\mathcal{E}^{1 / 2}(t) t},
\end{aligned}
$$

where the conductivity $\sigma_{0}$ is assumed constant within the radius $r_{c}(t)=\zeta_{c} r_{0}(t)$ of the current channel. Solving for $\mathcal{E}\left(t_{0}\right)$ one obtains

$$
\mathcal{E}\left(t_{0}\right)=\left(\frac{\rho_{0}}{\left(4 \pi \sigma_{0} C^{2}(\gamma) \zeta_{c}^{2} / 3\right)^{2}}\right)^{1 / 3}\left(2 \int_{0}^{t_{0}} \frac{I^{2}(t) d t}{t}\right)^{2 / 3} .
$$

The radial position of the current channel is given by

$$
\begin{aligned}
r_{c}(t) & =\zeta_{c} C(\gamma)\left(\frac{\mathcal{E}(t)}{\rho_{0}}\right)^{1 / 4} t^{1 / 2} \\
& =\left(\frac{3 C^{4}(\gamma) \zeta_{c}^{4}}{4 \pi \rho_{0} \sigma_{0}}\right)^{1 / 6}\left(2 \int_{0}^{t} \frac{I^{2}\left(t^{\prime}\right) d t^{\prime}}{t^{\prime}}\right)^{1 / 6} t^{1 / 2} .
\end{aligned}
$$

For a short pulse with a power law rise $I(t)=I_{0}\left(t / t_{0}\right)^{\alpha}$ and a sudden drop, it follows that

$$
r_{c}(t)=\left(\frac{3 C^{4}(\gamma) \xi_{c}^{4}}{4 \pi \alpha \rho_{0} \sigma_{0}}\right)^{1 / 6} I_{0}^{1 / 3} t^{1 / 2}
$$

which corresponds to the expression obtained by Braginskii for the spark channel radius. ${ }^{16}$ From numerical computations Braginskii concluded that the channel radius is weakly dependent on the current rise time, which is consistent with the $\alpha^{-1 / 6}$ dependence mentioned previously. Using the values assumed by Braginskii, that is, $\alpha=1, \rho_{0}=1.29 \mathrm{~kg} / \mathrm{m}^{3}$, and $\sigma_{0}$ $=2.225 \times 10^{4} \mathrm{~S} / \mathrm{m}$, the constant that defines the channel radius is $\zeta_{c}=0.526$ so that $r_{c}(t)=0.093 I_{0}^{1 / 3} t^{1 / 2}$. This radial position is represented in Fig. 5 by the central vertical line. One verifies that the pressure inside the channel is approximately constant and the velocity is proportional to the radius. Assuming that the temperature rise is concentrated in a thin shell at the position $r_{c}$ and that the temperature inside the channel is approximately constant one essentially recovers Braginskii's model.

Now, assuming a current pulse with a simple linear rise and exponential drop given by $I(t)=I_{0}\left(t / t_{0}\right) \exp \left(1-t / t_{0}\right)$, one calculates $(\epsilon=\exp (1))$

$$
2 \int_{0}^{t} \frac{I^{2}(t) d t}{t}=\frac{\epsilon^{2} I_{0}^{2}}{2}\left[1-\left(1+\frac{2 t}{t_{0}}\right) \exp \left(-\frac{2 t}{t_{0}}\right)\right],
$$

obtaining the energy input estimate at current peak 


$$
\mathcal{E}_{0}=\left(\frac{\epsilon^{2}-3}{2}\right)^{2 / 3} \mathcal{E}_{B}=1.69 \mathcal{E}_{B}
$$

and the total energy input estimate

$$
\mathcal{E}_{\infty}=\left(\frac{\epsilon}{\sqrt{2}}\right)^{4 / 3} \mathcal{E}_{B}=2.39 \mathcal{E}_{B},
$$

where

$$
\mathcal{E}_{B}=\left(\frac{\rho_{0} I_{0}^{4}}{\left[4 \pi \sigma_{0} C^{2}(\gamma) \zeta_{c}^{2} / 3\right]^{2}}\right)^{1 / 3}
$$

is the Braginskii energy. These energy estimates approximately agree with Plooster's results ${ }^{28}$ for a spark discharge in air. Taking into account the loss of energy by radiation, it is reasonable to take the energy at current peak $\mathcal{E}_{0}$ as an input for driving the shock wave from a lightning discharge. Although the theory of strong cylindrical shocks presupposes an almost instantaneous energy deposition, the introduction of an energy source with a finite pulse in the channel is not entirely inconsistent, since the channel can be assumed as a piston that drives the shock wave in a self-similar manner on the surrounding air. In general, the position of the current channel during the self-similar stage $\left(t_{0}<t<\tau_{0}, \tau_{0} \gg t_{0}\right)$ is given by

$$
r_{c}(t)=\zeta_{c} C(\gamma)\left(\frac{\mathcal{E}_{0}}{\rho_{0}}\right)^{1 / 4} t^{1 / 2}
$$

Finally, at pressure equilibration on axis, the temperature on the edge of the channel is

$$
\frac{T_{\mathrm{eq}}}{T_{0}}=\frac{T\left(r_{c}, t_{\mathrm{eq}}\right)}{T_{0}}=\gamma M^{2}\left(t_{\mathrm{eq}}\right) \hat{T}\left(\zeta_{c}\right)=\frac{\hat{T}\left(\zeta_{c}\right)}{\hat{p}(0)}=12.6
$$

with $\hat{T}\left(\zeta_{c}\right)=\hat{p}\left(\zeta_{c}\right) / \hat{\rho}\left(\zeta_{c}\right)=3.93\left(\hat{p}\left(\zeta_{c}\right)=0.314, \quad \hat{\rho}\left(\zeta_{c}\right)=0.0798\right.$ and $\hat{v}\left(\zeta_{c}\right)=0.376$ for $\gamma=7 / 5$ ). For ambient temperature the corresponding temperature on the edge is $T_{\mathrm{eq}} \simeq 3800 \mathrm{~K}$ when $p\left(0, t_{\mathrm{eq}}\right)=p_{0}$. The temperature across the boundary increases rapidly to values between 8000 and $10000 \mathrm{~K}$ inside the channel during the decay of the return current in a lightning stroke. $^{12}$

\section{APPENDIX C: AIR BACKFLOW AND COMPRESSION WAVE}

After the pressure in the lightning channel falls to atmospheric pressure, the air in the shock wave region continues to expand by inertia forming a region of rarefaction. Eventually, the negative pressure drop stops the expansion and the air begins to contract into the channel.

By analyzing the results of several numerical computations and some limited experimental results, Hill ${ }^{31}$ estimated the channel radius at pressure equilibration as $R_{H} \cong 0.2$. Since Hill uses a characteristic radius that is twice the characteristic radius defined by Plooster, ${ }^{27}$ one has $R_{H}=\lambda_{P} / 2$. Now, according to the results of Appendix B, the channel radius is related to the shock radius by $r_{c}(t)=\zeta_{c} r_{0}(t)$ $=0.526 r_{0}(t)$. Using the value $r_{0}\left(t_{\mathrm{eq}}\right)=0.787 R_{0}$ obtained in Appendix A for pressure equilibration on axis, one obtains $r_{c}\left(t_{\mathrm{eq}}\right)=0.414 R_{0}$ corresponding to $R_{H}=0.173$, which agrees with Hill's rough estimate, though out of the strong shock regime. After pressure equilibration, the compression region across the shock wave is followed by a rarefaction zone. The minimum pressure on axis is reached when the position of the shock wave nearly equals the characteristic radius $R_{0}$ defined in Appendix A, which corresponds to $\lambda_{P}=0.838$ in Plooster's coordinates. This point indicates the onset of backflow at the channel, which occurs for $\tau_{P}=c_{0} t_{b} / R_{P} \cong 0.44 \mathrm{ac}-$ cording to Plooster's results ${ }^{27}$ and corresponds to $t_{b} / \tau_{0}$ $=\left(R_{P} / R_{0}\right) c_{0} t_{b} / R_{P} \cong 0.53$. In a second analysis of numerical results, Hill ${ }^{14}$ displays a set of curves and tables adapted from Plooster's results. ${ }^{27}$ From the curves one can estimate the maximum backflow velocity at the channel boundary as

$$
\frac{v_{m}}{c_{0}} \cong 0.013 \text {. }
$$

Also, the backflow acceleration at the compression wave front can be derived from Hill's tables and written in a form appropriate to the present analysis:

$$
a_{0} \cong 0.093 \frac{\left(\gamma p_{0}\right)^{3 / 2}}{\rho_{0} \mathcal{E}^{1 / 2}} .
$$

Hill ${ }^{14}$ used the tabular values to study the Rayleigh-Taylor instability of a spark produced in the Naval Research Laboratory (NRL). ${ }^{32}$ The acceleration calculated in the case of the NRL spark is $a_{0} \cong 1.66 \times 10^{5} \mathrm{~m} / \mathrm{s}^{2}$ for $\mathcal{E} \cong 600 \mathrm{~J} / \mathrm{m}$.

The conditions of backflow can be represented by a simple compression wave according with the general solution ${ }^{26}$ :

$$
\begin{aligned}
& x=t\left(c_{0}+\frac{1}{2}(\gamma+1) v\right)+f(v), \\
& \rho(v)=\rho_{0}\left(1+\frac{\gamma-1}{2} \frac{v}{c_{0}}\right)^{2 /(\gamma-1)}, \\
& p(v)=p_{0}\left(1+\frac{\gamma-1}{2} \frac{v}{c_{0}}\right)^{2 \gamma /(\gamma-1)} .
\end{aligned}
$$

This solution corresponds to a plane traveling wave and only approximately applies to the cylindrical geometry of the present problem. The acceleration experienced by the gas is

$$
a=\frac{\partial v}{\partial t}=\frac{-v-2 c_{0} /(\gamma+1)}{t+[2 /(\gamma+1)] \partial f / \partial v},
$$

and the velocity gradient is

$$
\frac{\partial v}{\partial x}=\frac{2 /(\gamma+1)}{t+[2 /(\gamma+1)] \partial f / \partial v} .
$$

Hence, the overpressure in the compression wave is given by

$$
\Delta p=-\int_{c_{0} t}^{x_{m}} \rho(x, t) a(x, t) d x=-\int_{0}^{v_{m}} \rho(v) a(v, t)\left(\frac{\partial v}{\partial x}\right)^{-1} d v,
$$

which depends only on the value of the peak backflow velocity $v_{m}$ 


$$
\begin{aligned}
\Delta p= & \rho_{0}\left(\frac{\gamma+1}{2}\right) \int_{0}^{v_{m}}\left(1+\frac{\gamma-1}{2} \frac{v}{c_{0}}\right)^{2 /(\gamma-1)}\left(v+\frac{2 c_{0}}{\gamma+1}\right) d v \\
= & \rho_{0} c_{0}^{2}\left(\frac{\gamma-1}{\gamma(\gamma+1)}\right)\left[\left(1+\frac{\gamma-1}{2} \frac{v_{m}}{c_{0}}\right)^{(\gamma+1) /(\gamma-1)}\right. \\
& \left.\times\left(1+\frac{(\gamma+1)^{2}}{2(\gamma-1)} \frac{v_{m}}{c_{0}}\right)-1\right] \\
= & \rho_{0} c_{0} v_{m}\left(1+\frac{3+\gamma}{4} \frac{v_{m}}{c_{0}}+\frac{5+\gamma}{12} \frac{v_{m}^{2}}{c_{0}^{2}}+O\left(v_{m}^{3}\right)\right) . \quad(\mathrm{C} 7)
\end{aligned}
$$

Similarly, the average density in the compression wave front is

$$
\begin{aligned}
\bar{\rho} & =\frac{\rho_{0}}{v_{m}} \int_{0}^{v_{m}}\left(1+\frac{\gamma-1}{2} \frac{v}{c_{0}}\right)^{2 /(\gamma-1)} d v \\
& =\rho_{0}\left[\left(1+\frac{\gamma-1}{2} \frac{v_{m}}{c_{0}}\right)^{(\gamma+1) /(\gamma-1)}-1\right] \frac{2 c_{0}}{(\gamma+1) v_{m}} \\
& =\rho_{0}\left(1+\frac{1}{2} \frac{v_{m}}{c_{0}}+\frac{3-\gamma}{12} \frac{v_{m}^{2}}{c_{0}^{2}}+O\left(v_{m}^{3}\right)\right) .
\end{aligned}
$$

Therefore, according with Eq. (C1) for small peak velocity $v_{m} \ll c_{0}$

$$
\Delta p \cong \rho_{0} c_{0} v_{m} \text {, and } \bar{\rho} \cong \rho_{0} .
$$

Now, the acceleration gradient in the compression wave is determined from

$$
\begin{gathered}
\left(\frac{\gamma+1}{2}\right)\left(t+\frac{2}{\gamma+1} \frac{\partial f}{\partial v}\right)\left(v+\frac{2 c_{0}}{\gamma+1}\right) \frac{\partial a}{\partial x} \\
=-a\left[1+\left(v+\frac{2 c_{0}}{\gamma+1}\right) \frac{\partial^{2} f}{\partial v^{2}} \frac{\partial v}{\partial x}\right] \\
-(\gamma+1)\left(v+\frac{2 c_{0}}{\gamma+1}\right) \frac{\partial v}{\partial x},
\end{gathered}
$$

which yields the scale length of the acceleration profile

$$
\begin{aligned}
L_{a} & =\frac{a}{\partial a / \partial x} \\
& =-\frac{1}{a}\left(\frac{\gamma+1}{2}\right)\left(v+\frac{2 c_{0}}{\gamma+1}\right)^{2}\left(1+\frac{2 a}{\gamma+1} \frac{\partial^{2} f}{\partial v^{2}}\right)^{-1} .
\end{aligned}
$$

At the compression wave front $v=0$ and

$$
\begin{aligned}
& a_{0}=-c_{0}\left(\frac{\partial f}{\partial v}\right)_{0}^{-1}, \\
& \left(a L_{a}\right)_{0}=-c_{0}^{2}\left[\frac{\gamma+1}{2}+\left(a \frac{\partial^{2} f}{\partial v^{2}}\right)_{0}\right]^{-1} .
\end{aligned}
$$

The compression wave is driven by an imaginary piston related to the backflow of air. For a piston moving in an arbitrary manner with position and velocity given by $X(t)$ and $X^{\prime}(t)$, and initial conditions $X(0)=0$ and $X^{\prime}(0)=0$, respectively, the function $f(v)$ is determined by the boundary conditions at the surface of the piston:

$$
\begin{aligned}
& v=X^{\prime}(t), \\
& f(v)=X(t)-t\left(c_{0}+\frac{1}{2}(\gamma+1) X^{\prime}(t)\right) .
\end{aligned}
$$

Hence, the compression (or rarefaction) wave driven by the piston is described in terms of the parameter $\tau$ by

$$
\begin{aligned}
& v=X^{\prime}(\tau), \\
& x=X(\tau)+(t-\tau)\left(c_{0}+\frac{1}{2}(\gamma+1) X^{\prime}(\tau)\right) .
\end{aligned}
$$

The function $f$ and its derivatives can be written as

$$
\begin{aligned}
f & =X(\tau)-\tau\left(c_{0}+\frac{1}{2}(\gamma+1) X^{\prime}(\tau)\right) \\
\frac{\partial f}{\partial v} & =\frac{1}{d v / d \tau} \frac{d f}{d \tau} \\
& =\frac{1}{X^{\prime \prime}(\tau)}\left(-c_{0}-\frac{1}{2}(\gamma-1) X^{\prime}(\tau)-\frac{\tau}{2}(\gamma+1) X^{\prime \prime}(\tau)\right),
\end{aligned}
$$

$$
\begin{aligned}
\frac{\partial^{2} f}{\partial v^{2}} & =\frac{1}{(d v / d \tau)^{2}} \frac{d^{2} f}{d \tau^{2}}-\frac{1}{(d v / d \tau)^{3}} \frac{d^{2} v}{d \tau^{2}} \frac{d f}{d \tau} \\
& =\frac{1}{X^{\prime \prime}(\tau)}\left[-\gamma+\frac{X^{\prime \prime \prime}(\tau)}{\left[X^{\prime \prime}(\tau)\right]^{2}}\left(c_{0}+\frac{1}{2}(\gamma-1) X^{\prime}(\tau)\right)\right] .
\end{aligned}
$$

Applying the conditions at the compression wave front, namely, $x=0, \tau=0, v=0, X(0)=0, X^{\prime}(0)=0$, leads to

$$
\begin{aligned}
& f(0)=0, \\
& \left(\frac{\partial f}{\partial v}\right)_{0}=-\frac{c_{0}}{X^{\prime \prime}(0)}, \\
& \left(\frac{\partial^{2} f}{\partial v^{2}}\right)_{0}=\frac{1}{X^{\prime \prime}(0)}\left(-\gamma+\frac{c_{0} X^{\prime \prime \prime}(0)}{\left[X^{\prime \prime}(0)\right]^{2}}\right) .
\end{aligned}
$$

Then, the acceleration and the scale length of the acceleration profile at the compression wave front become

$$
\begin{aligned}
& a_{0}=X^{\prime \prime}(0), \\
& \left(L_{a}\right)_{0}=\frac{c_{0}^{2} / X^{\prime \prime}(0)}{(\gamma-1) / 2-c_{0} X^{\prime \prime \prime}(0) /\left[X^{\prime \prime}(0)\right]^{2}} .
\end{aligned}
$$

The values of the acceleration and its scale length depend on the piston motion. A reasonably approximation to the compression wave is obtained adopting an imaginary piston that accelerates initially according to a parabolic motion and then slowly accommodates in its final position: 


$$
\begin{aligned}
X(t) & =X_{0}\left[1-\left(1+\frac{t}{\sqrt{n} \tau_{m}}\right)^{n} \exp \left(-\frac{\sqrt{n} t}{\tau_{m}}\right)\right] \\
& =X_{0}\left(\frac{1}{2} \frac{t^{2}}{\tau_{m}^{2}}-\frac{1}{3 \sqrt{n}} \frac{t^{3}}{\tau_{m}^{3}}+O\left(t^{4}\right)\right) .
\end{aligned}
$$

The peak backflow velocity $v_{m}$ in the compression wave corresponds to

$$
\begin{aligned}
& v_{m}=X^{\prime}\left(\tau_{m}\right)=\left(\frac{1+\sqrt{n}}{\sqrt{n}}\right)^{n-1} \exp (-\sqrt{n}) \frac{X_{0}}{\tau_{m}}, \\
& X^{\prime \prime}\left(\tau_{m}\right)=0 .
\end{aligned}
$$

At the compression wave front one has

$$
\begin{aligned}
& X(0)=0, \\
& X^{\prime}(0)=0, \\
& X^{\prime \prime}(0)=X_{0} / \tau_{m}^{2}, \\
& X^{\prime \prime \prime}(0)=-2 X_{0} /\left(\sqrt{n} \tau_{m}^{3}\right) .
\end{aligned}
$$

These relations allow to calculate the acceleration and its scale length at the compression wave front in terms of the parameter $n$, the maximum backflow velocity $v_{m}$ and the time interval $\tau_{m}$ for the velocity to increase from zero to the peak value, after which the velocity falls off with the time constant $\tau_{m}$ (this time interval is of the order of the characteristic time $\left.\tau_{0}\right)$ :

$$
\begin{aligned}
& a_{0}=\frac{X_{0}}{\tau_{m}^{2}}=\left(\frac{\sqrt{n}}{1+\sqrt{n}}\right)^{n-1} \exp (\sqrt{n}) \frac{v_{m}}{\tau_{m}}, \\
& \left(L_{a}\right)_{0}=\frac{c_{0}^{2} \tau_{m}^{2} / X_{0}}{(\gamma-1) / 2+2 c_{0} \tau_{m} /\left(\sqrt{n} X_{0}\right)} \cong \frac{\sqrt{n}}{2} c_{0} \tau_{m} .
\end{aligned}
$$

This expression of the scale length was simplified using the condition $v_{m} \ll c_{0}$ introduced by Eq. (C1).

Using the results of Eqs. (C9) in the pressure balance condition $\Delta p=\bar{\rho}\left(a L_{a}\right)_{0}$, one obtains an equation for the parameter $n$ which gives compatible values of the average density, acceleration, and acceleration scale length at the compression wave front:

$$
\frac{\sqrt{n}}{2}\left(\frac{\sqrt{n}}{1+\sqrt{n}}\right)^{n-1} \exp (\sqrt{n})=1 .
$$

Hence

$$
\begin{aligned}
& n=0.326601, \\
& \begin{aligned}
a_{0} & =\frac{2}{\sqrt{n}}\left(\frac{v_{m}}{c_{0}}\right)\left(\frac{\tau_{0}}{\tau_{m}}\right) \frac{c_{0}^{2}}{R_{0}} \\
& =\frac{2}{\sqrt{n}}\left(\frac{\pi}{\gamma(\gamma-1)}\right)^{1 / 2}\left(\frac{v_{m}}{c_{0}}\right)\left(\frac{\tau_{0}}{\tau_{m}}\right) \frac{\left(\gamma p_{0}\right)^{3 / 2}}{\rho_{0} \mathcal{E}^{1 / 2}}, \\
\left(L_{a}\right)_{0} & \cong \frac{\sqrt{n}}{2}\left(\frac{\tau_{m}}{\tau_{0}}\right) R_{0} .
\end{aligned}
\end{aligned}
$$

Substituting the value of the peak backflow velocity $(\mathrm{C} 1)$ and comparing with the expression (C2) for the acceleration at the compression wavefront, it follows that

$$
\begin{aligned}
& \tau_{m} \cong 1.16 \tau_{0}, \\
& \left(L_{a}\right)_{0} \cong 0.331 R_{0} .
\end{aligned}
$$

Now, the position of the current channel at the onset of air backflow can be estimated by the difference between the characteristic radius $R_{0}$, which defines the position of balance between the excess energy and the thermal energy of air, and the acceleration scale length $L_{a}$ given by Eq. (C24):

$$
r_{c}\left(t_{b}\right) \cong R_{0}-\left(L_{a}\right)_{0} \cong 0.669 R_{0}=0.282\left(\frac{\mathcal{E}_{0}}{\gamma p_{0}}\right)^{1 / 2} .
$$

Using Eq. (C25) combined with Eqs. (B6) and (B7) the energy per unit volume required to form a hot channel in the atmosphere is estimated by

$$
\frac{\mathcal{E}_{\infty}}{\pi r_{c}^{2}\left(t_{b}\right)} \cong 0.80 \mathrm{MJ} / \mathrm{m}^{3} \text {. }
$$

Finally, an estimate of the density scale length across the channel boundary, during the backflow stage, can be made considering the equations of continuity and viscous motion with approximately constant pressure $p \cong p_{0}$, since, according with Eq. (C9), $\Delta p \ll p_{0}$ :

$$
\begin{aligned}
& \frac{d}{d x}(\rho v)=0, \\
& \frac{d}{d x}\left(\rho v^{2}-\frac{4 \eta}{3} \frac{d v}{d x}\right)=0 .
\end{aligned}
$$

These equations imply $\rho T \cong$ constant and the existence of an external heating source (Joule heating by the current flowing in the channel) to satisfy the energy balance equation. The solution is given by $\rho v \cong \rho_{0} v_{m} / 2$ in average and

$$
\frac{\rho(x)}{\rho_{0}}=\frac{1}{1+\exp \left(2 x / L_{\rho}\right)}
$$

with the scale length of the density profile determined by the mass flow across the viscous boundary:

$$
L_{\rho}=\left(\frac{\rho}{|d \rho / d x|}\right)_{0} \cong \frac{16 \eta}{3 \rho_{0} v_{m}} .
$$


${ }^{1}$ M. A. Uman, Lightning (Dover, New York, 1969).

${ }^{2}$ R. Fieux, C. Gary, and P. Hubert, Nature 257, 212 (1975).

${ }^{3}$ J. D. Barry, Ball Lightning and Bead Lightning (Plenum, New York, 1980).

${ }^{4}$ M. A. Uman, The Lightning Discharge (Academic, New York, 1987).

${ }^{5}$ R. J. Fisher, G. H. Schnetzer, R. Thottappillil, V. A. Rakov, M. A. Uman, and J. D. Goldberg, J. Geophys. Res. 98, 22887 (1993).

${ }^{6}$ V. A. Rakov, M. A. Uman, Lightning - Physics and Effects (Cambridge University Press, Cambridge, 2003), Chap. 20.

${ }^{7}$ M. M. F. Saba, O. Pinto, Jr., N. N. Solórzano, and A. Eybert-Berard, Atmos. Res. 76, 402 (2005).

${ }^{8}$ M. A. Uman, J. Atmos. Terr. Phys. 23, 43 (1962).

${ }^{9}$ M. A. Uman and R. E. Voshall, J. Geophys. Res. 73, 497 (1968).

${ }^{10}$ A. M. Boichenko, Plasma Phys. Rep. 22, 1012 (1996).

${ }^{11}$ G. K. Tumakaev, Tech. Phys. 40, 662 (1995).

${ }^{12}$ N. L. Aleksandrov, E. M. Bazelyan, and M. N. Shneider, Plasma Phys. Rep. 26, 893 (2000).

${ }^{13}$ G. O. Ludwig, M. M. F. Saba, and C. Potvin, Controlled Fusion and Plasma Physics (30th EPS Conference 2003), European Conference Abstracts Vol. 27A, CD-ROM file P-1.40.

${ }^{14}$ R. D. Hill, Phys. Fluids B 3, 1787 (1991).

${ }^{15}$ G. O. Ludwig, 2nd IAEA Technical Meeting on the Theory of Plasma Instabilities: Transport, Stability and their Interaction, Miramare, Trieste,
Italy, 2-4 March 2005 (IAEA, Vienna, 2005).

${ }^{16}$ S. I. Braginskii, Sov. Phys. JETP 7,1068 (1958).

${ }^{17}$ V. P. Idone, J. Geophys. Res. 100, 22943 (1995).

${ }^{18}$ D. L. Jones, G. G. Goyer, and M. N. Plooster, J. Geophys. Res. 73, 3121 (1968).

${ }^{19}$ L. I. Sedov, C. R. (Dokl.) Acad. Sci. URSS 52, 17 (1946).

${ }^{20}$ L. I. Sedov, Similarity and Dimensional Methods in Mechanics (Academic, New York, 1959).

${ }^{21}$ G. Taylor, Proc. R. Soc. London, Ser. A 201, 159 (1950).

${ }^{22}$ A. Sakurai, J. Phys. Soc. Jpn. 8, 662 (1953).

${ }^{23}$ S. C. Lin, J. Appl. Phys. 25, 54 (1954).

${ }^{24}$ A. Sakurai, in Basic Developments in Fluid Dynamics, edited by M. Holt (Academic, New York, 1965), Vol. 1.

${ }^{25}$ G. C. Vlases and D. L. Jones, Phys. Fluids 9, 478 (1966).

${ }^{26}$ L. D. Landau and E. M. Lifshitz, Fluid Mechanics (Pergamon, Oxford, 1959). Chap. X, Secs. 99 and 94.

${ }^{27}$ M. N. Plooster, Phys. Fluids 13, 2665 (1970).

${ }^{28}$ M. N. Plooster, Phys. Fluids 14, 2111 (1971).

${ }^{29}$ M. N. Plooster, Phys. Fluids 14, 2124 (1971).

${ }^{30}$ A. H. Paxton, R. L. Gardner, and L. Baker, Phys. Fluids 29, 2736 (1986).

${ }^{31}$ R. D. Hill, Phys. Fluids B 2, 3209 (1990).

${ }^{32}$ J. R. Greig, R. E. Pechacek, and M. Raleigh, Phys. Fluids 28, 2357 (1985). 